Mathematical Models and Methods in Applied Sciences

Vol. 29, No. 8 (2019) 1511-1552

(C) World Scientific Publishing Company

DOI: $10.1142 / \mathrm{S} 0218202519500271$

\title{
Approximate cloaking for electromagnetic waves via transformation optics: Cloaking versus infinite energy
}

\author{
Hoai-Minh Nguyen* and Loc Tran ${ }^{\dagger}$ \\ Department of Mathematics, EPFL SB CAMA, Station 8, \\ CH-1015 Lausanne, Switzerland \\ *hoai-minh.nguyen@epfl.ch \\ †loc.tran@epfl.ch
}

Received 10 January 2018

Revised 28 February 2019

Accepted 22 March 2019

Published 24 May 2019

Communicated by G. Allaire

\begin{abstract}
We study the approximate cloaking via transformation optics for electromagnetic waves in the time harmonic regime in which the cloaking device only consists of a layer constructed by the mapping technique. Due to the fact that no-lossy layer is required, resonance might appear and the analysis is delicate. We analyze both non-resonant and resonant cases. In particular, we show that the energy can blow up inside the cloaked region in the resonant case and/whereas cloaking is achieved in both cases. Moreover, the degree of visibility depends on the compatibility of the source inside the cloaked region and the system. These facts are new and distinct from known mathematical results in the literature.
\end{abstract}

Keywords: Electromagnetic scattering; Maxwells equations; invisibility cloaking; transformation optics; infinite energy.

AMS Subject Classification: 35A15, 35B40, 78A40, 78M30

\section{Introduction}

Cloaking via transformation optics was introduced by Pendry et al ${ }^{34}$ for the Maxwell system and by Leonhardt $\frac{26}{26}$ in the geometric optics setting. They used a singular change of variables which blows up a point into a cloaked region. The same transformation was used by Greenleaf et al $!^{16}$ to establish the non-uniqueness of Calderon's problem. The singular nature of the cloaks presents various difficulties in practice as well as in theory: (1) they are hard to fabricate and (2) in certain cases, the correct definition of the corresponding electromagnetic fields is not obvious. To avoid using the singular structure, various regularized schemes have been proposed. One of them was suggested by Kohn et al. in Ref. 24] in which they used

${ }^{*}$ Corresponding author 
a transformation which blows up a small ball of radius $\rho$ instead of a point into the cloaked region.

Approximate cloaking schemes for the Helmholtz equation based on the regularized transformations introduced in Ref. 24 have been studied extensively in the

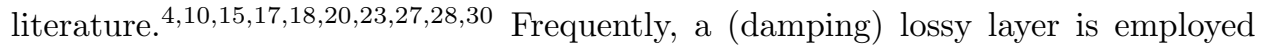
inside the transformation cloak. Without the lossy layer, the field inside the cloaked region might depend on the field outside, and resonance might appear and affect the cloaking ability of the cloak. ${ }^{28}$ Approximate cloaking was investigated in the time domain for the acoustic waves $31 \mid 32$ In Ref. 32 , the dependence of the material constants on frequency via the Drude-Lorentz model was taken into account.

Cloaking for electromagnetic waves via transformation optics has been mathematically investigated by several authors. Greenleaf et al ${ }^{[15}$ and Weder $\stackrel{3839}{{ }^{3}}$ studied cloaking for the singular scheme mentioned above by considering finite energy solutions. Concerning this approach, the information inside the cloaked region is not seen by observers outside. Approximate cloaking for the Maxwell equations using schemes in the spirit of Ref. 24] was considered in different works $\frac{57725}{25}$ Ammari et al $\stackrel{5}{5}$ investigated the cloaking problem where the cloaked object is placed inside the core of the cloaking device which is a cavity. Additional layers were used and designed in order to cancel the first-order scattering coefficients. Similar idea was used for the conducting problem where the layers are designed in an appropriate way to cancel the first high-order generalized polarization tensors. ${ }^{3}$ Bao et al. ${ }^{7}$ studied the approximate cloaking using a lossy layer inside the transformation cloak. Their approach is as follows. Taking into account the lossy layer, one easily obtains an estimate for the electric field inside the lossy layer. This estimate depends on the property of the lossy layer and degenerates as the lossy property disappears. They then used the equation of the electric field in the lossy layer to derive estimates for the electric field on the boundary of the lossy region in some negative Sobolev norm. The cloaking estimate can be finally deduced from the integral representation for the electric field. This approach essentially uses the property of the lossy layer and does not provide an optimal estimate of the degree of visibility in general. For example, when a fixed lossy layer is employed, they showed that the degree of visibility is of the order $\rho^{2}$, which is not optimal. In Ref. 25, Lassas and Zhou considered the transformation cloak in a symmetric setting, dealt with the non-resonant case (see Definition 2.2 and studied the limit of the solutions of the approximate cloaking problem near the cloak interface using separation of variables. Other regularized schemes are considered in Ref. 13 .

In this paper, we investigate approximate cloaking for the Maxwell equation in the time harmonic regime using a scheme in the spirit of Ref. 24. More precisely, we consider the situation where the cloaking device only consists of a layer constructed by the mapping technique and there is no source in that layer. Due to the fact that no-lossy (damping) layer is required, resonance might appear and the analysis is subtle. Our analysis is given in both non-resonant and resonant cases 
(Definition 2.2 and the results can be briefly summarized as follows:

(i) In the non-resonant case, cloaking is achieved, and the energy remains finite inside the cloaked region.

(ii) In the resonant case, cloaking is also achieved. Nevertheless, the degree of invisibility varies and depends on the compatibility of the source with the system (see (2.6) and (2.10). Moreover, the energy inside the cloaked region might explode in the incompatible case. See Theorems 2.2 and 2.3 .

(iii) The degree of visibility is of the order $\rho^{3}$ for both non-resonant and resonant cases if no source is inside the cloaked region (Theorems 2.1 and 2.2.

We also investigate the behavior of the field in the whole space (Theorems 2.1 2.3 and establish the optimality of the convergence rate (Sec. 5). Our results are new and distinct from the works mentioned above. First, cloaking takes place even if the energy explodes inside the cloaked region as $\delta$ goes to 0 . Second, in the resonant case with finite energy inside the cloaked region, the fields inside the cloaked region satisfy a non-local structure. Optimal estimates for the degree of visibility are derived for all cases. In particular, in the case of a fixed lossy layer (non-resonant case), the degree of visibility is of the order $\rho^{3}$ instead of $\rho^{2}$ as obtained previously. Both non-resonant and resonant cases are analyzed in detail without assuming the symmetry of the cloaking setting.

Our approach is different from the ones in the works mentioned. It is based on several subtle estimates for the effect of small inclusion involving the blowup structure. Part of the analysis is on Maxwell's equations in the low frequency regime, which is interesting in itself. The approach in this paper is inspired from Ref. 28 where the acoustic setting was considered. Nevertheless, the analysis for the electromagnetic setting is challenging and requires further new ideas due to the non-standard structure coming from the mapping technique and the complexity of electromagnetic structures/phenomena in comparison with acoustic ones. The Helmholtz decomposition and Stokes' theorem are involved in the Maxwell context.

\section{Statement of the Main Results}

In this section, we describe the problem in more detail and state the main results of this paper. For simplicity of notations, we suppose that the cloak occupies the annular region $B_{2} \backslash B_{1}$ and the cloaked region is the unit ball $B_{1}$ in $\mathbb{R}^{3}$ in which the permittivity and the permeability are given by two $3 \times 3$ matrices $\varepsilon, \mu$, respectively. Here and in what follows, for $r>0$, let $B_{r}$ denote the open ball in $\mathbb{R}^{3}$ centered at the origin and of radius $r$. Through this paper, we assume that

$$
\varepsilon, \mu \text { are real, symmetric, }
$$

and uniformly elliptic in $B_{1}$, i.e. for a.e. $x \in B_{1}$ and for some $\Lambda \geq 1$,

$$
\frac{1}{\Lambda}|\xi|^{2} \leq\langle\varepsilon(x) \xi, \xi\rangle, \quad\langle\mu(x) \xi, \xi\rangle \leq \Lambda|\xi|^{2} \quad \text { for all } \xi \in \mathbb{R}^{3} .
$$


We assume in addition that $\varepsilon, \mu$ are piecewise $C^{1}$ in order to ensure the wellposedness of Maxwell's equations in the frequency domain (via the unique continuation principle). In the spirit of the scheme in Ref. 24, the permittivity and permeability of the cloaking region are given by

$$
\left(\varepsilon_{c}, \mu_{c}\right):=\left(F_{\rho_{*}} I, F_{\rho_{*}} I\right) \text { in } B_{2} \backslash B_{1},
$$

where $F_{\rho}: \mathbb{R}^{3} \rightarrow \mathbb{R}^{3}$ with $\rho \in(0,1 / 2)$ is defined by

$$
F_{\rho}= \begin{cases}x & \text { in } \mathbb{R}^{3} \backslash B_{2}, \\ \left(\frac{2-2 \rho}{2-\rho}+\frac{|x|}{2-\rho}\right) \frac{x}{|x|} & \text { in } B_{2} \backslash B_{\rho}, \\ \frac{x}{\rho} & \text { in } B_{\rho} .\end{cases}
$$

We denote

$$
F_{0}(x)=\lim _{\rho \rightarrow 0} F_{\rho}(x) \quad \text { for } x \in \mathbb{R}^{3} .
$$

As usual, for a matrix $A \in \mathbb{R}^{3 \times 3}$ and for a bi-Lipschitz homeomorphism $T$, the following notation is used:

$$
T_{*} A(y)=\frac{D T(x) A(x) D T^{T}(x)}{|\operatorname{det} D T(x)|} \quad \text { with } y=T(x) .
$$

Assume that the medium is homogeneous outside the cloak and the cloaked region. In the presence of the cloaked object and the cloaking device, the medium in the whole space $\mathbb{R}^{3}$ is given by $\left(\varepsilon_{c}, \mu_{c}\right)$ which is defined as follows:

$$
\left(\varepsilon_{c}, \mu_{c}\right)= \begin{cases}(I, I) & \text { in } \mathbb{R}^{3} \backslash B_{2}, \\ \left(F_{\rho_{*}} I, F_{\rho_{*}} I\right) & \text { in } B_{2} \backslash B_{1}, \\ (\varepsilon, \mu) & \text { in } B_{1} .\end{cases}
$$

With the cloak and the object, in the time harmonic regime of frequency $\omega>0$, the electromagnetic field generated by current $J \in\left[L^{2}\left(\mathbb{R}^{3}\right)\right]^{3}$ with compact support is the unique (Silver-Müller) radiating solution $\left(E_{c}, H_{c}\right) \in\left[H_{\text {loc }}\left(\operatorname{curl}, \mathbb{R}^{3}\right)\right]^{2}$ of the system

$$
\begin{cases}\nabla \times E_{c}=i \omega \mu_{c} H_{c} & \text { in } \mathbb{R}^{3}, \\ \nabla \times H_{c}=-i \omega \varepsilon_{c} E_{c}+J & \text { in } \mathbb{R}^{3} .\end{cases}
$$

For an open subset $U$ of $\mathbb{R}^{3}$, denote

$$
\begin{aligned}
H(\operatorname{curl}, U) & :=\left\{\phi \in\left[L^{2}(U)\right]^{3} ; \nabla \times \phi \in\left[L^{2}(U)\right]^{3}\right\}, \\
H_{\mathrm{loc}}(\operatorname{curl}, U) & :=\left\{\phi \in\left[L_{\mathrm{loc}}^{2}(U)\right]^{3} ; \nabla \times \phi \in\left[L_{\mathrm{loc}}^{2}(U)\right]^{3}\right\},
\end{aligned}
$$

and

$$
\|\phi\|_{H(\operatorname{curl}, U)}:=\|\phi\|_{L^{2}(U)}+\|\nabla \times \phi\|_{L^{2}(U)} .
$$


Recall that, for $\omega>0$, a solution $(E, H) \in\left[H_{\text {loc }}\left(\operatorname{curl}, \mathbb{R}^{3} \backslash B_{R}\right)\right]^{2}$, for some $R>0$, of the Maxwell equations

$$
\begin{cases}\nabla \times E=i \omega H & \text { in } \mathbb{R}^{3} \backslash B_{R}, \\ \nabla \times H=-i \omega E & \text { in } \mathbb{R}^{3} \backslash B_{R}\end{cases}
$$

is called radiating if it satisfies one of the (Silver-Müller) radiation conditions

$$
H \times x-|x| E=O(1 /|x|) \quad \text { and } \quad E \times x+|x| H=O(1 /|x|) \quad \text { as }|x| \rightarrow+\infty .
$$

Here and in what follows, for $\alpha \in \mathbb{R}, O\left(|x|^{\alpha}\right)$ denotes a quantity whose norm is bounded by $C|x|^{\alpha}$ for some constant $C>0$.

Denote $J_{\text {ext }}$ and $J_{\text {int }}$ the restriction of $J$ into $\mathbb{R}^{3} \backslash B_{1}$ and $B_{1}$, respectively. It is clear that

$$
J= \begin{cases}J_{\text {ext }} & \text { in } \mathbb{R}^{3} \backslash B_{1}, \\ J_{\text {int }} & \text { in } B_{1} .\end{cases}
$$

In the homogeneous medium (without the cloaking device and the cloaked object), the electromagnetic field generated by $J_{\text {ext }}$ is the unique (Silver-Müller) radiating solution $(E, H) \in\left[H_{\text {loc }}\left(\operatorname{curl}, \mathbb{R}^{3}\right)\right]^{2}$ to the system

$$
\begin{cases}\nabla \times E=i \omega H & \text { in } \mathbb{R}^{3}, \\ \nabla \times H=-i \omega E+J_{\text {ext }} & \text { in } \mathbb{R}^{3} .\end{cases}
$$

We next introduce the functional space $\mathcal{N}$ which is related to the notion of resonance and plays a role in our analysis.

Definition 2.1. Let $D$ be a smooth bounded subset of $\mathbb{R}^{3}$ such that $\mathbb{R}^{3} \backslash D$ is connected. Set

$$
\mathcal{N}(D):=\left\{(\mathbf{E}, \mathbf{H}) \in[H(\operatorname{curl}, D)]^{2}:(\mathbf{E}, \mathbf{H}) \text { satisfies the system } 2.2\right\},
$$

where

$$
\begin{cases}\nabla \times \mathbf{E}=i \omega \mu \mathbf{H} & \text { in } D, \\ \nabla \times \mathbf{H}=-i \omega \varepsilon \mathbf{E} & \text { in } D, \\ \nabla \times \mathbf{E} \cdot \nu=\nabla \times \mathbf{H} \cdot \nu=0 & \text { on } \partial D .\end{cases}
$$

In the case $D=B_{1}$, we simply denote $\mathcal{N}\left(B_{1}\right)$ by $\mathcal{N}$.

The resonant and non-resonant notions are defined as follows.

Definition 2.2. The cloaking system 2.1 is said to be non-resonant if $\mathcal{N}=$ $\{(0,0)\}$. Otherwise, the cloaking system 2.1 is called resonant.

Remark 2.1. The definition of resonant and non-resonant notions of the cloaking system is related to an eigenvalue problem of a compact and self-adjoint operator (see Lemma 4.4 and its proof). In fact, set

$$
\mathbb{V}=\{\varphi \in H(\operatorname{curl}, D): \operatorname{div}(\varepsilon \varphi)=0, \varepsilon \varphi \cdot \nu=0 \text { on } \partial D, \nabla \times \varphi \cdot \nu=0 \text { on } \partial D\}
$$


and let $A: \mathbb{V} \rightarrow \mathbb{V}$ be defined by

$$
\langle A E, \varphi\rangle_{\langle\mathbb{V}, \mathbb{V}\rangle}=-2 \int_{D} \varepsilon E \cdot \bar{\varphi} d x \quad \text { for all } \varphi \in \mathbb{V} .
$$

One can check that $A$ is compact and self-adjoint. Moreover, the cloaking system (2.1) is resonant if and only if (see 4.29)

$$
\omega^{-1} E+A E=0 \text { has a non-zero solution in } \mathbb{V}
$$

with $D=B_{1}$. Since $\langle A E, E\rangle_{\langle\mathbb{V}, \mathbb{V}\rangle}=-2 \int_{D} \varepsilon E \cdot \bar{E} \leq 0$ for $E \in \mathbb{V}$, it follows that $A$ is injective and there exists a countably infinite set $K \subset \mathbb{R} \backslash\{0\}$ such that the system is resonant if and only if $\omega \in K$. When $D$ is radially symmetric, $\varepsilon=\mu=I$, the resonant characterization is given in Lemma 5.1 .

Our main result in the non-resonant case is the following theorem.

Theorem 2.1. Let $\rho \in(0,1 / 2), R_{0}>2$, and let $J \in L^{2}\left(\mathbb{R}^{3}\right)$ be such that supp $J_{\text {ext }} \subset \subset B_{R_{0}} \backslash B_{2}$. Assume that system (2.1) is non-resonant. We have, for all $K \subset \subset \mathbb{R}^{3} \backslash \bar{B}_{1}$,

$$
\begin{aligned}
& \left\|\left(F_{\rho}^{-1} * E_{c}, F_{\rho}^{-1} * H_{c}\right)-(E, H)\right\|_{H(\operatorname{curl}, K)} \\
& \quad \leq C\left(\rho^{3}\left\|J_{\text {ext }}\right\|_{L^{2}\left(B_{R_{0}} \backslash B_{2}\right)}+\rho^{2}\left\|J_{\text {int }}\right\|_{L^{2}\left(B_{1}\right)}\right)
\end{aligned}
$$

for some positive constant $C$ depending only on $R_{0}, \omega, K, \mu, \varepsilon$. Moreover,

$$
\lim _{\rho \rightarrow 0}\left(E_{c}, H_{c}\right)=C l\left(0, J_{\text {int }}\right) \quad \text { in }\left[H\left(\operatorname{curl}, B_{1}\right)\right]^{2},
$$

where $C l\left(0, J_{\text {int }}\right)$ is defined in Definition 2.3 .

Here and in what follows, one denotes

$$
F * E=\left(D F^{-T} E\right) \circ F^{-1}
$$

for an appropriate bijective map $F$ and a vector field $E$.

The notation $C l(\cdot, \cdot)$ used in Theorem 2.1 is defined as follows.

Definition 2.3. Assume that $\mathcal{N}=\{(0,0)\}$. Let $\theta_{1}, \theta_{2} \in\left[L^{2}\left(B_{1}\right)\right]^{3}$. Define $C l\left(\theta_{1}, \theta_{2}\right)=\left(E_{0}, H_{0}\right)$, where $\left(E_{0}, H_{0}\right) \in\left[H\left(\operatorname{curl}, B_{1}\right)\right]^{2}$ is the unique solution to the system

$$
\begin{cases}\nabla \times E_{0}=i \omega \mu H_{0}+\theta_{1} & \text { in } B_{1}, \\ \nabla \times H_{0}=-i \omega \varepsilon E_{0}+\theta_{2} & \text { in } B_{1}, \\ \nabla \times E_{0} \cdot \nu=\nabla \times H_{0} \cdot \nu=0 & \text { on } \partial B_{1} .\end{cases}
$$

Remark 2.2. The existence and the uniqueness of $\left(E_{0}, H_{0}\right)$ are established in Lemma 4.4

Remark 2.3. In Ref. 39, the conditions

$$
\nabla \times\left. E_{0} \cdot \nu\right|_{\text {int }}=\nabla \times\left. H_{0} \cdot \nu\right|_{\text {int }}=0
$$


are also imposed on the boundary of the cloaked region. This is different from Ref. 15] (see also p. 459 of Ref. 25), where the following boundary conditions are imposed for solutions satisfying some integrability conditions, which are called finite energy solutions,

$$
E_{0} \times\left.\nu\right|_{\text {int }}=H_{0} \times\left.\nu\right|_{\text {int }}=0 .
$$

The novelty of Theorem 2.1 relies on the fact that no lossy layer is required. The result holds for a general class of pairs $(\varepsilon, \mu)$. Applying Theorem 2.1 to the case where a fixed lossy layer is used, one obtains that the degree of visibility is of the order $\rho^{3}$ which is better than $\rho^{2}$ as established previously ${ }^{7}$ for the case $J_{\text {int }} \equiv 0$. In contrast with Refs. [5, 7] and 13, the estimate of visibility is considered up to the cloaked region and the behavior of the electromagnetic fields are also established inside the cloaked region in Theorem 2.1.

We next consider the resonant case. We begin with the compatible case, i.e. (2.6) holds.

Theorem 2.2. Let $\rho \in(0,1 / 2), R_{0}>2$, and $J \in\left[L^{2}\left(\mathbb{R}^{3}\right)\right]^{3}$ be such that supp $J_{\text {ext }} \subset \subset B_{R_{0}} \backslash B_{2}$. Assume that system (2.1) is resonant and the following compatibility condition holds:

$$
\int_{B_{1}} J_{\mathrm{int}} \cdot \overline{\boldsymbol{E}} d x=0 \quad \text { for all }(\boldsymbol{E}, \boldsymbol{H}) \in \mathcal{N} .
$$

We have, for all $K \subset \subset \mathbb{R}^{3} \backslash \bar{B}_{1}$,

$$
\begin{aligned}
& \left\|\left(F_{\rho}^{-1} * E_{c}, F_{\rho}^{-1} * H_{c}\right)-(E, H)\right\|_{H(\operatorname{curl}, K)} \\
& \quad \leq C\left(\rho^{3}\left\|J_{\mathrm{ext}}\right\|_{L^{2}\left(B_{R_{0}} \backslash B_{2}\right)}+\rho^{2}\left\|J_{\mathrm{int}}\right\|_{L^{2}\left(B_{1}\right)}\right)
\end{aligned}
$$

for some positive constant $C$ depending only on $R_{0}, \omega, K, \mu$, and $\varepsilon$. Moreover,

$$
\lim _{\rho \rightarrow 0}\left(E_{c}, H_{c}\right)=C l\left(0, J_{\text {int }}\right) \quad \text { in }\left[H\left(\operatorname{curl}, B_{1}\right)\right]^{2},
$$

where $C l\left(0, J_{\text {int }}\right)$ is defined in Definition 2.4 .

In Theorem 2.2, we use the following notion.

Definition 2.4. Assume that $\mathcal{N} \neq\{(0,0)\}$. Let $\theta_{1}, \theta_{2} \in\left[L^{2}\left(B_{1}\right)\right]^{3}$ be such that

$$
\int_{B_{1}}\left(\theta_{2} \cdot \overline{\mathbf{E}}-\theta_{1} \cdot \overline{\mathbf{H}}\right) d x=0 \quad \text { for all }(\mathbf{E}, \mathbf{H}) \in \mathcal{N} \text {. }
$$

Let $\left(E_{0}, H_{0}, E^{\perp}, H^{\perp}\right) \in\left[H_{\text {loc }}\left(\operatorname{curl}, \mathbb{R}^{3}\right)\right]^{2} \times \mathcal{N}^{\perp}$ be the unique solution of the following systems:

$$
\left\{\begin{array} { l l } 
{ \nabla \times E _ { 0 } = \nabla \times H _ { 0 } = 0 } & { \text { in } \mathbb { R } ^ { 3 } \backslash B _ { 1 } , } \\
{ \operatorname { d i v } E _ { 0 } = \operatorname { d i v } H _ { 0 } = 0 } & { \text { in } \mathbb { R } ^ { 3 } \backslash B _ { 1 } , } \\
{ \nabla \times E _ { 0 } = i \omega \mu H _ { 0 } + \theta _ { 1 } } & { \text { in } B _ { 1 } , } \\
{ \nabla \times H _ { 0 } = - i \omega \varepsilon E _ { 0 } + \theta _ { 2 } } & { \text { in } B _ { 1 } , }
\end{array} \quad \text { and } \quad \left\{\begin{array}{ll}
\nabla \times E^{\perp}=i \omega \mu H^{\perp} & \text { in } B_{1}, \\
\nabla \times H^{\perp}=-i \omega \varepsilon E^{\perp} & \text { in } B_{1}, \\
\varepsilon E^{\perp} \cdot \nu=\left.E_{0} \cdot \nu\right|_{\text {ext }} & \text { on } \partial B_{1}, \\
\mu H^{\perp} \cdot \nu=\left.H_{0} \cdot \nu\right|_{\text {ext }} & \text { on } \partial B_{1}
\end{array}\right.\right.
$$


such that

$$
\left|\left(E_{0}(x), H_{0}(x)\right)\right|=O\left(|x|^{-2}\right) \quad \text { for large }|x| .
$$

Denote $C l\left(\theta_{1}, \theta_{2}\right)$ the restriction of $\left(E_{0}, H_{0}\right)$ in $B_{1}$.

Here and in what follows, $\mathcal{N}(D)^{\perp}$ denotes the orthogonal space of $\mathcal{N}(D)$ with respect to the standard scalar product in $\left[L^{2}(D)\right]^{6}$. The uniqueness and the existence of $\left(E_{0}, H_{0}, E^{\perp}, H^{\perp}\right)$ are given in Lemmas 4.5 and 4.6 .

In Definition 2.4 $\left(E_{0}, H_{0}\right)$ is determined by a non-local structure 2.9. This is new to our knowledge.

In the incompatible case, we have the following.

Theorem 2.3. Let $\rho \in(0,1 / 2), R_{0}>2$, and $J \in\left[L^{2}\left(\mathbb{R}^{3}\right)\right]^{3}$ be such that supp $J_{\text {ext }} \subset \subset B_{R_{0}} \backslash B_{2}$. Assume that system (2.1) is resonant and the compatibility condition does not hold, i.e.

$$
\int_{B_{1}} J_{\mathrm{int}} \cdot \overline{\boldsymbol{E}} d x \neq 0 \quad \text { for some }(\boldsymbol{E}, \boldsymbol{H}) \in \mathcal{N} .
$$

We have, for all $K \subset \subset \mathbb{R}^{3} \backslash \bar{B}_{1}$,

$$
\begin{aligned}
& \left(F_{\rho}^{-1} * E_{c}, F_{\rho}^{-1} * H_{c}\right)-(E, H) \|_{H(\operatorname{curl}, K)} \\
& \quad \leq C\left(\rho^{3}\left\|J_{\text {ext }}\right\|_{L^{2}\left(B_{R_{0}} \backslash B_{2}\right)}+\rho\left\|J_{\mathrm{int}}\right\|_{L^{2}\left(B_{1}\right)}\right)
\end{aligned}
$$

and

$$
\liminf _{\rho \rightarrow 0} \rho\left\|\left(E_{c}, H_{c}\right)\right\|_{L^{2}\left(B_{1}\right)}>0 .
$$

Some comments on Theorems 2.2 and 2.3 are in order. Theorems 2.2 and 2.3 imply in particular that cloaking is achieved even in the resonant case. Moreover, without any source in the cloaked region, one can achieve the same degree of visibility as in the non-resonant case considered in Theorem 2.1. Nevertheless, the degree of visibility varies and depends on the compatibility of the source inside the cloaked region. More precisely, the rate of the convergence of $\left(E_{c}, H_{c}\right)-(E, H)$ outside $\bar{B}_{1}$ in the compatible case is of the order $\rho^{2}$ which is better than the incompatible resonant case where an estimate of the order $\rho$ is obtained. The rate of the convergence is optimal and discussed in Sec. 5. By 2.12 , the energy inside the cloaked region blows up at least with the rate $1 / \rho$ as $\rho \rightarrow 0$ in the incompatible case.

We now describe briefly the ideas of the proofs of Theorems $2.1,2.3$. Set

$$
\left(\mathcal{E}_{\rho}, \mathcal{H}_{\rho}\right)=\left(F_{\rho}^{-1} * E_{c}, F_{\rho}^{-1} * H_{c}\right) \quad \text { in } \mathbb{R}^{3} .
$$

It follows from a standard change of variables formula (see e.g. Lemma 3.9 that $\left(\mathcal{E}_{\rho}, \mathcal{H}_{\rho}\right) \in\left[H_{\text {loc }}\left(\operatorname{curl}, \mathbb{R}^{3}\right)\right]^{2}$ is the unique (Silver-Müller) radiating solution to

$$
\begin{cases}\nabla \times \mathcal{E}_{\rho}=i \omega \mu_{\rho} \mathcal{H}_{\rho} & \text { in } \mathbb{R}^{3}, \\ \nabla \times \mathcal{H}_{\rho}=-i \omega \varepsilon_{\rho} \mathcal{E}_{\rho}+J_{\rho} & \text { in } \mathbb{R}^{3}\end{cases}
$$


where

$$
\left(\varepsilon_{\rho}, \mu_{\rho}\right)=\left(F_{\rho}^{-1} * \varepsilon_{c}, F_{\rho}^{-1} * \mu_{c}\right)= \begin{cases}(I, I) & \text { in } \mathbb{R}^{3} \backslash B_{\rho}, \\ \left(\rho^{-1} \varepsilon(\cdot / \rho), \rho^{-1} \mu(\cdot / \rho)\right) & \text { in } B_{\rho},\end{cases}
$$

and

$$
J_{\rho}= \begin{cases}J_{\text {ext }} & \text { in } \mathbb{R}^{3} \backslash B_{2}, \\ \rho^{-2} J_{\text {int }}(\cdot / \rho) & \text { in } B_{\rho} \\ 0 & \text { otherwise. }\end{cases}
$$

We can then derive Theorems $2.1,2.3$ by studying the difference between $\left(\mathcal{E}_{\rho}, \mathcal{H}_{\rho}\right)$ and $(E, H)$ in $\mathbb{R}^{3} \backslash B_{1}$ and the behavior of $\left(\mathcal{E}_{\rho}, \mathcal{H}_{\rho}\right)(\rho \cdot)$ in $B_{1}$. It is well known that when material parameters inside a small inclusion are bounded from below and above by positive constants, the effect of the small inclusion is small $6[37$ Without this assumption, the effect of the inclusion might not be small ${ }^{24} 29$ unless there is an appropriate lossy layer $\sqrt[5 / 13]{ }$ In our setting, the boundedness assumption is violated (see (2.14) ) and no lossy layer is used. Nevertheless, the effect of the small inclusion is still small due to the special structure induced from (2.14).

It is worth noting that system (2.5), which involves in the resonant and nonresonant definitions, and the condition of compatibility 2.6 , appears very naturally in our context. Indeed, note that if $\left(E_{c}, H_{c}\right)$ is bounded in $\left[H\left(\operatorname{curl}, B_{1}\right)\right]^{2}$, one can check that, up to a subsequence, $\left(\rho \mathcal{E}_{\rho}, \rho \mathcal{H}_{\rho}\right)(\rho \cdot)=\left(E_{c}, H_{c}\right)$ converges weakly in $\left[H\left(\operatorname{curl}, B_{1}\right)\right]^{2}$ to $\left(E_{0}, H_{0}\right)$ which satisfies system $(2.5)$ with $\left(\theta_{1}, \theta_{2}\right)=(0, J)$.

The paper is organized as follows. In Sec. 3, we establish some basic facts and recall some known results related to Maxwell's equations. These materials will be used in the proofs of Theorems 2.1 2.3. The proofs of Theorems 2.1 2.3 are given in Sec. 4. Finally, in Sec. 5, we discuss the optimality of the convergence rate in Theorems 2.12 .3 .

\section{Preliminaries}

In this section, we establish some basic facts and recall some known results related to Maxwell's equations that will be repeatedly used in the proofs of Theorems 2.1. 2.3 In what follows in this section, $D$ denotes a smooth, bounded, open subset of $\mathbb{R}^{3}$ and on its boundary, $\nu$ denotes its normal unit vector directed to the exterior. We begin with a variant of the classic Stokes' theorem for an exterior domain.

Lemma 3.1. Assume that $\mathbb{R}^{3} \backslash D$ is simply connected and let $u \in H_{\text {loc }}\left(\operatorname{curl}, \mathbb{R}^{3} \backslash D\right)$ be such that

$$
\nabla \times u=0 \quad \text { in } \mathbb{R}^{3} \backslash D \quad \text { and } \quad|u(x)|=O\left(|x|^{-2}\right) \quad \text { for large }|x| .
$$

There exists $\xi \in H_{\mathrm{loc}}^{1}\left(\mathbb{R}^{3} \backslash D\right)$ such that

$$
\nabla \xi=u \quad \text { in } \mathbb{R}^{3} \backslash D \quad \text { and } \quad|\xi(x)|=O\left(|x|^{-1}\right) \quad \text { for large }|x| .
$$


Proof. By Theorem 2.9 of Ref. 14, there exists $\eta_{n} \in H^{1}\left(B_{n} \backslash D\right)$ for large $n$ such that

$$
\nabla \eta_{n}=u \quad \text { in } B_{n} \backslash D \text { and } \quad \int_{\partial B_{2}} \eta_{n}=0 .
$$

It follows that, for $m>n$ large,

$$
\eta_{m}=\eta_{n} \quad \text { in } B_{n} \backslash D .
$$

Let $\eta$ be the limit of $\eta_{n}$ as $n \rightarrow+\infty$. Then $\eta \in H_{\text {loc }}^{1}\left(\mathbb{R}^{3} \backslash D\right)$ and

$$
\nabla \eta=u \quad \text { in } \mathbb{R}^{3} \backslash D \text {. }
$$

Fix $x, y \in \mathbb{R}^{3}$ large enough with $|y|>|x|$ and denote $\hat{x}=x /|x|$ and $\hat{y}=y /|y|$. Using (3.1), we have, by the fundamental theorem of calculus,

$$
|\eta(x)-\eta(y)| \leq|\eta(|y| \hat{y})-\eta(|y| \hat{x})|+|\eta(|y| \hat{x})-\eta(|x| \hat{x})| \leq \frac{C}{|y|}+\int_{|x|}^{|y|} \frac{C}{|r|^{2}} d r
$$

for some positive constant $C$ independent of $x$ and $y$. It follows that

$$
|\eta(x)-\eta(y)| \leq \frac{C}{|y|}+\frac{C}{|x|}
$$

Hence $\lim _{|x| \rightarrow \infty} \eta(x)$ exists. Denote this limit by $\eta_{\infty}$. By letting $|y| \rightarrow+\infty$ in 3.2 , we obtain

$$
\left|\eta(x)-\eta_{\infty}\right| \leq \frac{C}{|x|} \quad \text { for }|x| \text { large enough. }
$$

The conclusion follows with $\xi=\eta-\eta_{\infty}$.

Let $U$ be a smooth open subset of $\mathbb{R}^{3}$. Denote

$$
H(\operatorname{div}, U):=\left\{\phi \in\left[L^{2}(U)\right]^{3}: \operatorname{div} \phi \in L^{2}(U)\right\} .
$$

Concerning a divergence free field in a bounded domain, one has the following result which is related to Stokes' theorem, see e.g. Theorems 3.4 and 3.6 of Ref. 14,

Lemma 3.2. Assume that $D$ is simply connected and let $u \in H(\operatorname{div}, D)$ be such that

$$
\operatorname{div} u=0 \quad \text { in } D \quad \text { and } \quad \int_{\Gamma_{i}} u \cdot \nu=0 \text { for all connected component } \Gamma_{i} \text { of } \partial D .
$$

There exists $\phi \in\left[H^{1}(D)\right]^{3}$ such that

$$
\nabla \times \phi=u \text { in } D \quad \text { and } \quad \operatorname{div} \phi=0 \text { in } D .
$$

Assume in addition that $u \cdot \nu=0$ on $\partial D$. Then $\phi$ can be chosen such that $\phi \times \nu=0 \quad$ on $\partial D$ and $\int_{\Gamma_{i}} \phi \cdot \nu=0$ for all connected component $\Gamma_{i}$ of $\partial D$. 
Moreover, such a $\phi$ is unique and, for some positive constant $C$,

$$
\|\phi\|_{H^{1}(D)} \leq C\|u\|_{L^{2}(D)} .
$$

The following result is a type of Helmholtz decomposition. It is a variant of Corollary 3.4 of Ref. 14, where $\sigma$ is a positive constant.

Lemma 3.3. Assume that $D$ is simply connected and let $\sigma$ be $a 3 \times 3$ uniformly elliptic matrix-valued function defined in D. For any $v \in\left[L^{2}(D)\right]^{3}$, there exist $p \in H^{1}(D)$ and $\phi \in\left[H^{1}(D)\right]^{3}$ such that

$$
v=\sigma \nabla p+\nabla \times \phi \quad \text { in } D, \quad \operatorname{div} \phi=0 \quad \text { in } D \quad \text { and } \quad \phi \times \nu=0 \quad \text { on } \partial D .
$$

Moreover,

$$
\|p\|_{H^{1}(D)}+\|\phi\|_{H^{1}(D)} \leq C\|v\|_{L^{2}(D)} .
$$

Proof. The proof given here is in the spirit of Ref. 14 as follows. By Lax-Milgram's theorem, there exists a unique solution $p \in H^{1}(D)$ with $\int_{D} p d x=0$ to the equation

$$
\int_{D} \sigma \nabla p \cdot \nabla q d x=\int_{D} v \cdot \nabla q d x \quad \text { for all } q \in H^{1}(D) .
$$

Moreover,

$$
\|p\|_{H^{1}(D)} \leq C\|v\|_{L^{2}(D)} .
$$

Then

$$
\operatorname{div}(v-\sigma \nabla p)=0 \quad \text { in } D \quad \text { and } \quad(v-\sigma \nabla p) \cdot \nu=0 \quad \text { on } \partial D .
$$

By Lemma 3.2, there exists $\phi \in\left[H^{1}(D)\right]^{3}$ such that

$$
\left\{\begin{array}{ll}
\nabla \times \phi=v-\sigma \nabla p & \text { in } D, \\
\operatorname{div} \phi=0 & \text { in } D, \\
\phi \times \nu=0 & \text { on } \partial D,
\end{array} \text { and }\|\phi\|_{H^{1}(D)} \leq C\|v-\sigma \nabla p\|_{L^{2}(D)} .\right.
$$

Combining (3.3), 3.4), and (3.5), we reach the conclusion for such a pair $(p, \phi)$.

We next present two standard lemmas concerning the uniqueness of the exterior problems for electrostatic settings, see e.g. Theorems 2 and 3 of Ref. 35 (see also Chap. 1 of Ref. 14). They are used in the study of the exterior problems in the low frequency regime. The first one, whose proof can be derived from Lemma 3.1, is as follows.

Lemma 3.4. Assume that $\mathbb{R}^{3} \backslash D$ is simply connected. Let $u \in H_{\text {loc }}\left(\operatorname{curl}, \mathbb{R}^{3} \backslash D\right) \cap$ $H_{\text {loc }}\left(\operatorname{div}, \mathbb{R}^{3} \backslash D\right)$ be such that

$$
\begin{cases}\nabla \times u=0 & \text { in } \mathbb{R}^{3} \backslash D \\ \operatorname{div} u=0 & \text { in } \mathbb{R}^{3} \backslash D \\ u \cdot \nu=0 & \text { on } \partial D\end{cases}
$$


and

$$
|u(x)|=O\left(|x|^{-2}\right) \quad \text { for large }|x|
$$

Then $u=0$ in $\mathbb{R}^{3} \backslash D$.

The second lemma, whose proof can be derived from Lemma 3.2 , is as follows.

Lemma 3.5. Assume that $\mathbb{R}^{3} \backslash D$ is simply connected and $u \in H_{\text {loc }}\left(\operatorname{curl}, \mathbb{R}^{3} \backslash D\right) \cap$ $H_{\text {loc }}\left(\operatorname{div}, \mathbb{R}^{3} \backslash D\right)$ is such that

$$
\left\{\begin{array}{ll}
\nabla \times u=0 & \text { in } \mathbb{R}^{3} \backslash D, \\
\operatorname{div} u=0 & \text { in } \mathbb{R}^{3} \backslash D, \\
u \times \nu=0 & \text { on } \partial D,
\end{array} \quad \int_{\Gamma_{i}} u \cdot \nu=0 \quad \text { for all connected component } \Gamma_{i} \text { of } \partial D,\right.
$$

and

$$
|u(x)|=O\left(|x|^{-2}\right) \quad \text { for large }|x| .
$$

Then $u=0$ in $\mathbb{R}^{3} \backslash D$.

The following result is a consequence of the Stratton-Chu formula.

Lemma 3.6. Let $0<k \leq k_{0}$. Assume that $D \subset \subset B_{1}$ and $(E, H) \in$ $\left[H_{\text {loc }}\left(\text { curl, } \mathbb{R}^{3} \backslash D\right)\right]^{2}$ is a radiating solution to the Maxwell equations

$$
\left\{\begin{array}{l}
\nabla \times E=i k H \quad \text { in } \mathbb{R}^{3} \backslash \bar{D}, \\
\nabla \times H=-i k E \quad \text { in } \mathbb{R}^{3} \backslash \bar{D} .
\end{array}\right.
$$

We have

$$
|(E(x), H(x))| \leq \frac{C}{|x|^{2}}(1+k|x|)\|(E, H)\|_{L^{2}\left(B_{3} \backslash D\right)} \quad \text { for }|x|>3
$$

for some positive constant $C$ independent of $x$ and $k$.

Proof. Set

$$
G_{k}(x, y)=\frac{e^{i k|x-y|}}{4 \pi|x-y|} \quad \text { for } x, \quad y \in \mathbb{R}^{3}, x \neq y .
$$

It is known that, see e.g. Theorem 6.6 and (6.10) in Ref. 11, the following variant of the Stratton-Chu formula holds, for $x \in \mathbb{R}^{3} \backslash \bar{D}$,

$$
\begin{aligned}
E(x)= & \nabla_{x} \times \int_{\partial B_{2}} \nu(y) \times E(y) G_{k}(x, y) d y+i k \int_{\partial B_{2}} \nu(y) \times H(y) G_{k}(x, y) d y \\
& -\nabla_{x} \int_{\partial B_{2}} \nu(y) \cdot E(y) G_{k}(x, y) d y
\end{aligned}
$$

Using the facts

$$
\left|\nabla G_{k}(x, y)\right| \leq \frac{C}{|x|^{2}}(1+k|x|) \quad \text { for } y \in \partial B_{2}, \quad x \in \mathbb{R}^{3} \backslash B_{3}
$$


and, since $\Delta E+k^{2} E=0$ in $\mathbb{R}^{3} \backslash D$,

$$
\begin{aligned}
& \|E\|_{L^{\infty}\left(\partial B_{2}\right)} \\
& \quad \leq C\|E\|_{L^{2}\left(B_{3} \backslash D\right)}, \quad \text { for some positive constant } C \text { depending only on } k_{0},
\end{aligned}
$$

we derive from 3.6 that

$$
|E(x)| \leq \frac{C}{|x|^{2}}(1+k|x|)\|(E, H)\|_{L^{2}\left(B_{3} \backslash D\right)} \quad \text { for }|x|>3 .
$$

Similarly, we obtain

$$
|H(x)| \leq \frac{C}{|x|^{2}}(1+k|x|)\|(E, H)\|_{L^{2}\left(B_{3} \backslash D\right)} \quad \text { for }|x|>3 .
$$

The conclusion now follows from 3.7 and 3.8 .

We next recall the following compactness results related to $H(\operatorname{curl}, \cdot)$ and $H(\operatorname{div}, \cdot)$.

Lemma 3.7. Let $\epsilon$ be a measurable symmetric uniformly elliptic matrix-valued function defined in D. Assume that one of the following two conditions holds:

(i) $\left(u_{n}\right)_{n \in \mathbb{N}} \subset H(\operatorname{curl}, D)$ is a bounded sequence in $H(\operatorname{curl}, D)$ such that

$\left(\operatorname{div}\left(\epsilon u_{n}\right)\right)_{n \in \mathbb{N}}$ converges in $H^{-1}(D)$ and $\left(u_{n} \times \nu\right)_{n \in \mathbb{N}}$ converges in $H^{-1 / 2}(\partial D)$.

(ii) $\left(u_{n}\right)_{n \in \mathbb{N}} \subset H(\operatorname{curl}, D)$ is a bounded sequence in $H(\operatorname{curl}, D)$ such that

$\left(\operatorname{div}\left(\epsilon u_{n}\right)\right)_{n \in \mathbb{N}}$ is bounded in $L^{2}(D)$ and $\left(\epsilon u_{n} \cdot \nu\right)_{n \in \mathbb{N}}$ converges in $H^{-1 / 2}(\partial D)$.

There exists a subsequence of $\left(u_{n}\right)_{n \in \mathbb{N}}$ which converges in $\left[L^{2}(D)\right]^{3}$.

The conclusion of Lemma 3.7 under condition (i) is Lemma 1 of Ref. 29 and has its roots in Refs. 19 and 12, The conclusion of Lemma 3.7 under condition (ii) can be obtained in the same way. These compactness results play a similar role as the compact embedding of $H^{1}$ into $L^{2}$ in the acoustic setting and are basic ingredients in our approach.

The following trace results related to $H(\operatorname{curl}, \cdot)$ and $H(\operatorname{div}, \cdot)$ are standard $\frac{19}{14}$

Lemma 3.8. Set $\Gamma=\partial D$. We have

$$
\|v \times \nu\|_{H^{-1 / 2}\left(\operatorname{div}_{\Gamma}, \Gamma\right)} \leq C\|v\|_{H(\operatorname{curl}, D)} \quad \text { for } v \in H(\operatorname{curl}, D)
$$

$$
\|v \cdot \nu\|_{H^{-1 / 2}(\Gamma)} \leq C\|v\|_{H(\operatorname{div}, D)} \quad \text { for } v \in H(\operatorname{div}, D) .
$$


Moreover, for any $h \in H^{-1 / 2}\left(\operatorname{div}_{\Gamma}, \partial D\right)$, there exists $\phi \in H(\operatorname{curl}, D)$ such that

$$
\phi \times \nu=h \quad \text { on } \partial D \quad \text { and } \quad\|\phi\|_{H(\operatorname{curl}, D)} \leq C\|h\|_{H^{-1 / 2}\left(\operatorname{div}_{\Gamma}, \partial D\right)} .
$$

Here $C$ denotes a positive constant depending only on D.

Here and in what follows, we denote

$$
\begin{aligned}
H^{-1 / 2}\left(\operatorname{div}_{\Gamma}, \Gamma\right) & :=\left\{\phi \in\left[H^{-1 / 2}(\Gamma)\right]^{3} ; \phi \cdot \nu=0 \text { and } \operatorname{div}_{\Gamma} \phi \in H^{-1 / 2}(\Gamma)\right\}, \\
\|\phi\|_{H^{-1 / 2}\left(\operatorname{div}_{\Gamma}, \Gamma\right)} & :=\|\phi\|_{H^{-1 / 2}(\Gamma)}+\left\|\operatorname{div}_{\Gamma} \phi\right\|_{H^{-1 / 2}(\Gamma)} .
\end{aligned}
$$

We finally recall the following change of variables for the Maxwell equations. It is the basic ingredient for cloaking using transformation optics for electromagnetic fields.

Lemma 3.9. Let $D, D^{\prime}$ be two open bounded connected subsets of $\mathbb{R}^{3}$ and $F: D \rightarrow$ $D^{\prime}$ be a bijective map such that $F \in C^{1}(\bar{D}), F^{-1} \in C^{1}\left(\bar{D}^{\prime}\right)$. Let $\varepsilon, \mu \in\left[L^{\infty}(D)\right]^{3 \times 3}$ and $j \in\left[L^{2}(D)\right]^{3}$. Assume that $(E, H) \in[H(\operatorname{curl}, D)]^{2}$ is a solution of the Maxwell equations

$$
\begin{cases}\nabla \times E=i \omega \mu H & \text { in } D, \\ \nabla \times H=-i \omega \varepsilon E+j & \text { in } D .\end{cases}
$$

Set, in $D^{\prime}$,

$$
E^{\prime}:=F * E:=\left(D F^{-T} E\right) \circ F^{-1} \quad \text { and } \quad H^{\prime}:=F * H:=\left(D F^{-T} H\right) \circ F^{-1} .
$$

Then $\left(E^{\prime}, H^{\prime}\right) \in\left[H\left(\operatorname{curl}, D^{\prime}\right)\right]^{2}$ satisfies

$$
\begin{cases}\nabla \times E^{\prime}=i \omega \mu^{\prime} H^{\prime} & \text { in } D^{\prime}, \\ \nabla \times H^{\prime}=-i \omega \varepsilon^{\prime} E^{\prime}+j^{\prime} & \text { in } D^{\prime},\end{cases}
$$

where

$$
\begin{gathered}
\varepsilon^{\prime}:=F_{*} \varepsilon:=\frac{D F \varepsilon D F^{T}}{|\operatorname{det} F|} \circ F^{-1}, \quad \mu^{\prime}:=F_{*} \mu:=\frac{D F \mu D F^{T}}{|\operatorname{det} F|} \circ F^{-1}, \\
\text { and } j^{\prime}:=F_{*} j=\frac{D F j}{|\operatorname{det} F|} \circ F^{-1} .
\end{gathered}
$$

Remark 3.1. It is worth noting the difference of $F *$ in the definition of $E^{\prime}$ and $H^{\prime}$, and $F_{*}$ in the definition of $\varepsilon^{\prime}, \mu^{\prime}$, and $j^{\prime}$.

\section{Proofs of the Main Results}

This section is devoted to the proof of Theorems 2.1 2.3 and is organized as follows. In Sec. 4.1, we establish various results related to $\left(\mathcal{E}_{\rho}, \mathcal{H}_{\rho}\right)$. The proof of Theorem 2.1 is given in Sec. 4.2 and the ones of Theorems 2.2 and 2.3 are given in Sec. 4.3 . 


\subsection{Some useful lemmas}

In this section, $D \subset B_{1}$ denotes a smooth open bounded subset of $\mathbb{R}^{3}$, and $\varepsilon$ and $\mu$ denote two $3 \times 3$ matrices defined in $D$ which are both assumed to be real, symmetric, and uniformly elliptic in $D$. We also assume that $D$ and $\mathbb{R}^{3} \backslash D$ are simply connected and $\varepsilon, \mu$ are piecewise $C^{1}$. The following lemma provides the stability of the exterior problem in the low frequency regime.

Lemma 4.1. Let $0<\rho<\rho_{0}$ and let $\left(E_{\rho}, H_{\rho}\right) \in\left[H_{\mathrm{loc}}\left(\operatorname{curl}, \mathbb{R}^{3} \backslash D\right)\right]^{2}$ be a radiating solution to the system

$$
\begin{cases}\nabla \times E_{\rho}=i \rho H_{\rho} & \text { in } \mathbb{R}^{3} \backslash D, \\ \nabla \times H_{\rho}=-i \rho E_{\rho} & \text { in } \mathbb{R}^{3} \backslash D .\end{cases}
$$

We have, for $R>1$,

$$
\left\|\left(E_{\rho}, H_{\rho}\right)\right\|_{H\left(\operatorname{curl}, B_{R} \backslash D\right)} \leq C_{R}\left(\left\|E_{\rho} \times \nu\right\|_{H^{-1 / 2}(\partial D)}+\left\|H_{\rho} \cdot \nu\right\|_{H^{-1 / 2}(\partial D)}\right)
$$

and

$$
\left\|\left(E_{\rho}, H_{\rho}\right)\right\|_{H\left(\operatorname{curl}, B_{R} \backslash D\right)} \leq C_{R}\left(\left\|E_{\rho} \times \nu\right\|_{H^{-1 / 2}(\partial D)}+\left\|H_{\rho} \times \nu\right\|_{H^{-1 / 2}(\partial D)}\right)
$$

for some positive constant $C_{R}$ depending only on $\rho_{0}, D$, and $R$.

Proof. Lemma 4.1 might be known but we cannot find a reference for it, see e.g. Refs. 2 and 36 and references therein for related results. For the convenience of the reader, we present its proof. We begin with the proof of 4.2 . Since $\left(E_{\rho}, H_{\rho}\right)$ satisfies 4.1, it suffices to prove that

$$
\left\|\left(E_{\rho}, H_{\rho}\right)\right\|_{L^{2}\left(B_{R} \backslash D\right)} \leq C_{R}\left(\left\|E_{\rho} \times \nu\right\|_{H^{-1 / 2}(\partial D)}+\left\|H_{\rho} \cdot \nu\right\|_{H^{-1 / 2}(\partial D)}\right)
$$

for $R>3$. Fixing $R>3$, we prove 4.4 by contradiction. Suppose that there exist a sequence $\left(\rho_{n}\right)_{n \in \mathbb{N}} \subset\left(0, \rho_{0}\right)$ and a sequence of radiating solutions $\left(\left(E_{n}, H_{n}\right)\right)_{n \in \mathbb{N}} \subset$ $\left[H\left(\operatorname{curl}, \mathbb{R}^{3} \backslash D\right)\right]^{2}$ of the system

$$
\begin{cases}\nabla \times E_{n}=i \rho_{n} H_{n} & \text { in } \mathbb{R}^{3} \backslash D, \\ \nabla \times H_{n}=-i \rho_{n} E_{n} & \text { in } \mathbb{R}^{3} \backslash D,\end{cases}
$$

such that

$$
\left\|\left(E_{n}, H_{n}\right)\right\|_{L^{2}\left(B_{R} \backslash D\right)}=1 \quad \text { for } n \in \mathbb{N}
$$

and

$$
\lim _{n \rightarrow 0}\left(\left\|E_{n} \times \nu\right\|_{H^{-1 / 2}(\partial D)}+\left\|H_{n} \cdot \nu\right\|_{H^{-1 / 2}(\partial D)}\right)=0 .
$$

Without loss of generality, one might assume that $\rho_{n} \rightarrow \rho_{\infty}$ as $n \rightarrow \infty$ for some $\rho_{\infty} \in\left[0, \rho_{0}\right]$. We only consider the case $\rho_{\infty}=0$. The case $\rho_{\infty}>0$ can be proven similarly. From 4.5 and 4.6, we have

$$
\left\|\left(E_{n}, H_{n}\right)\right\|_{H\left(\operatorname{curl}, B_{R} \backslash D\right)} \leq C .
$$


Here and in what follows in this proof, $C$ and $C_{r}$ denote positive constants independent of $n$. Applying Lemma 3.6, we obtain

$$
\left\|\left(E_{n}, H_{n}\right)\right\|_{H\left(\operatorname{curl}, B_{r} \backslash D\right)} \leq C_{r}
$$

for $r>3$. Since

$$
\Delta E_{\rho}+\rho^{2} E_{\rho}=\Delta H_{\rho}+\rho^{2} H_{\rho}=0 \quad \text { in } \mathbb{R}^{3} \backslash D,
$$

it follows from 4.7) that, for $r>3$,

$$
\left\|\left(E_{n}, H_{n}\right)\right\|_{H^{1}\left(B_{r+1} \backslash B_{r-1}\right)} \leq C_{r} .
$$

By the trace theory, we have

$$
\left\|\left(E_{n}, H_{n}\right)\right\|_{H^{1 / 2}\left(\partial B_{r}\right)} \leq C_{r} .
$$

Since the embedding of $H^{1 / 2}\left(\partial B_{r}\right)$ into $H^{-1 / 2}\left(\partial B_{r}\right)$ is compact, by applying (i) of Lemma 3.7 to $\left(E_{n}\right)$ and by applying (ii) of Lemma 3.7 to $\left(H_{n}\right)$, without loss of generality, one might assume that $\left(E_{n}, H_{n}\right)$ converges in $\left[L_{\text {loc }}^{2}\left(\mathbb{R}^{3} \backslash D\right)\right]^{6}$. Moreover, the limit $(E, H) \in\left[H_{\text {loc }}\left(\mathbb{R}^{3} \backslash D\right)\right]^{2}$ satisfies

$$
\left\{\begin{array} { l l } 
{ \nabla \times H = 0 } & { \text { in } \mathbb { R } ^ { 3 } \backslash D , } \\
{ \operatorname { d i v } H = 0 } & { \text { in } \mathbb { R } ^ { 3 } \backslash D , } \\
{ H \cdot \nu = 0 } & { \text { on } \partial D , }
\end{array} \text { and } \quad \left\{\begin{array}{ll}
\nabla \times E=0 & \text { in } \mathbb{R}^{3} \backslash D, \\
\operatorname{div} E=0 & \text { in } \mathbb{R}^{3} \backslash D, \\
E \times \nu=0 & \text { on } \partial D
\end{array}\right.\right.
$$

Applying Lemma 3.6 to $\left(E_{n}, H_{n}\right)$ and letting $n \rightarrow+\infty\left(\rho_{n} \rightarrow 0\right)$, we have

$$
|(E(x), H(x))|=O\left(|x|^{-2}\right) \quad \text { for large }|x| \text {. }
$$

On the other hand, since $E_{n}=-\frac{1}{i \rho_{n}} \nabla \times H_{n}$ in $\mathbb{R}^{3} \backslash D$, we have

$$
\int_{\Gamma_{i}} E_{n} \cdot \nu=0 \text { for all connected component } \Gamma_{i} \text { of } \partial D .
$$

Since $\left(E_{n}\right)$ converges to $E$ in $\left[L_{\text {loc }}^{2}\left(\mathbb{R}^{3} \backslash D\right)\right]^{3}$ and $\operatorname{div} E_{n}=\operatorname{div} E=0$ in $\mathbb{R}^{3} \backslash D$, it follows that $\left(E_{n}\right)$ converges to $E$ in $H_{\text {loc }}\left(\operatorname{div}, \mathbb{R}^{3} \backslash D\right)$. This in turn implies, by 4.10 ,

$$
\int_{\Gamma_{i}} E \cdot \nu=0 \text { for all connected component } \Gamma_{i} \text { of } \partial D \text {. }
$$

Applying Lemma 3.4 to $H$, we derive from 4.8 and 4.9 that

$$
H=0 \quad \text { in } \mathbb{R}^{3} \backslash D .
$$

Similarly, applying Lemma 3.5 to $E$, from 4.8, 4.9), and 4.11, we obtain ${ }^{\text {a }}$

$$
E=0 \quad \text { in } \mathbb{R}^{3} \backslash D \text {. }
$$

a When $\rho_{\infty}>0$, instead of being a solution of 4.8, $(E, H)$ is the radiating solution of 4.1 with $\rho=\rho_{\infty}$ and $E \times \nu=0$ on $\partial D$. This also implies that $(E, H)=(0,0)$ in $\mathbb{R}^{3} \backslash D$. 
From 4.6, 4.12, and 4.13 and the fact that $\left(E_{n}, H_{n}\right)$ converges to $(E, H)$ in $L_{\text {loc }}^{2}\left(\mathbb{R}^{3} \backslash D\right)$, we reach a contradiction. The proof of $\sqrt{4.2}$ is complete.

We next deal with 4.3 . The proof of 4.3 is similar to the one of 4.2 . However, instead of obtaining 4.8 and 4.11), we have

$$
\left\{\begin{array} { l l } 
{ \nabla \times H = 0 } & { \text { in } \mathbb { R } ^ { 3 } \backslash D , } \\
{ \operatorname { d i v } H = 0 } & { \text { in } \mathbb { R } ^ { 3 } \backslash D , } \\
{ H \times \nu = 0 } & { \text { on } \partial D , }
\end{array} \text { and } \quad \left\{\begin{array}{ll}
\nabla \times E=0 & \text { in } \mathbb{R}^{3} \backslash D, \\
\operatorname{div} E=0 & \text { in } \mathbb{R}^{3} \backslash D, \\
E \times \nu=0 & \text { on } \partial D,
\end{array}\right.\right.
$$

and

$$
\int_{\Gamma} H \cdot \nu=\int_{\Gamma} E \cdot \nu=0 \text { for all connected component } \Gamma \text { of } \partial D \text {. }
$$

By the same arguments, we can derive that $(E, H)=(0,0)$ in $\mathbb{R}^{3}$, which also yields a contradiction. The details are left to the reader.

Remark 4.1. We have

$$
\operatorname{div}_{\Gamma}\left(E_{\rho} \times \nu\right)=\nabla \times E_{\rho} \cdot \nu=i \rho H_{\rho} \cdot \nu \quad \text { on } \partial D .
$$

It follows that

$$
\begin{aligned}
\left\|E_{\rho} \times \nu\right\|_{H^{-1 / 2}\left(\operatorname{div}_{\Gamma}, \partial D\right)} & \leq\left\|E_{\rho} \times \nu\right\|_{H^{-1 / 2}(\partial D)}+\left\|H_{\rho} \cdot \nu\right\|_{H^{-1 / 2}(\partial D)} \\
& \leq \frac{1}{\rho}\left\|E_{\rho} \times \nu\right\|_{H^{-1 / 2}\left(\operatorname{div}_{\Gamma}, \partial D\right)} .
\end{aligned}
$$

The next lemma gives an estimate for solutions of Maxwell's equations in the low frequency regime, which in turn implies an estimate for the effect of a small inclusion after a change of variables.

Lemma 4.2. Let $0<\rho<1 / 2, R>1 / 2$, and let $\left(E_{\rho}, H_{\rho}\right) \in\left[H_{\mathrm{loc}}\left(\operatorname{curl}, \mathbb{R}^{3} \backslash D\right)\right]^{2}$ be a radiating solution to the system

$$
\begin{cases}\nabla \times E_{\rho}=i \omega \rho H_{\rho} & \text { in } \mathbb{R}^{3} \backslash D, \\ \nabla \times H_{\rho}=-i \omega \rho E_{\rho} & \text { in } \mathbb{R}^{3} \backslash D .\end{cases}
$$

We have

$$
\left|\left(E_{\rho}(x), H_{\rho}(x)\right)\right| \leq C \rho^{3}\left\|\left(E_{\rho}, H_{\rho}\right)\right\|_{L^{2}\left(B_{2} \backslash D\right)} \quad \text { for } x \in B_{3 R / \rho} \backslash B_{2 R / \rho}
$$

for some constant $C$ depending only $R$.

Lemma 4.2 is well known, see e.g. Ref. 22, It can be derived from Stratton-Chu's formula and Stoke's theorem (see also Eq. (12) of Ref. 22).

The following compactness result is used in the proof of Theorems 2.1 2.3 .

Lemma 4.3. Let $\left(\left(E_{n}, H_{n}\right)\right)_{n}$ be a bounded sequence in $[H(\operatorname{curl}, D)]^{2}$ and let $\left(\left(\theta_{1, n}, \theta_{2, n}\right)\right)_{n}$ be a convergent sequence in $\left[L^{2}(D)\right]^{6}$. Assume that

$$
\begin{cases}\nabla \times E_{n}=i \mu H_{n}+\theta_{1, n} & \text { in } D, \\ \nabla \times H_{n}=-i \varepsilon E_{n}+\theta_{2, n} & \text { in } D,\end{cases}
$$


and

$$
\left(\left(\nabla \times E_{n} \cdot \nu, \nabla \times H_{n} \cdot \nu\right)\right)_{n} \quad \text { converges in }\left[H^{-1 / 2}(\partial D)\right]^{2} .
$$

Then, up to a subsequence, $\left(\left(E_{n}, H_{n}\right)\right)_{n}$ converges in $[H(\operatorname{curl}, D)]^{2}$.

Remark 4.2. A comparison with Lemma 3.7 is necessary. The difference between Lemma 4.3 and part (i) Lemma 3.7 is that the sequence $\left(E_{n} \times \nu\right)_{n}$ or $(H \times \nu)_{n}$ is not required to be convergent in $H^{-1 / 2}(\partial D)$. The difference between Lemma 4.3 and part (ii) Lemma 3.7 is that the sequence $\left(\operatorname{div}\left(\varepsilon E_{n}\right)\right)_{n}$ or $\left(\operatorname{div}\left(\mu H_{n}\right)\right)_{n}$ is not required to be bounded in $L^{2}(D)$. Nevertheless, in Lemma 4.3 (4.14 is assumed.

Proof. It suffices to prove that, up to a subsequence, $\left(\left(E_{n}, H_{n}\right)\right)_{n}$ converges in $\left[L^{2}(D)\right]^{6}$. By Lemma 3.3 , there exist $\left(q_{n}\right)_{n} \subset H^{1}(D)$ and $\left(\phi_{n}\right)_{n} \subset\left[H^{1}(D)\right]^{3}$ such that, for all $n$,

$$
\varepsilon E_{n}=\varepsilon \nabla q_{n}+\nabla \times \phi_{n} \quad \text { in } \mathrm{D}, \quad \operatorname{div} \phi_{n}=0 \quad \text { in } D, \quad \text { and } \quad \phi_{n} \times \nu=0 \quad \text { on } \partial D .
$$

Moreover, we have

$$
\left\|q_{n}\right\|_{H^{1}(D)}+\left\|\phi_{n}\right\|_{\left[H^{1}(D)\right]^{3}} \leq C\left\|E_{n}\right\|_{L^{2}(D)} \leq C
$$

for some positive constant $C$ independent of $n$. From (4.17), without loss of generality, one might assume that

$$
\left(q_{n}\right)_{n} \text { and }\left(\phi_{n}\right)_{n} \text { converge in } L^{2}(D) \text { and }\left[L^{2}(D)\right]^{3} \text {, respectively. }
$$

From 4.16 and an integration by parts, we derive that, for all $n$,

$$
\int_{D} \varepsilon \nabla q_{n} \cdot \nabla p d x=\int_{D} \varepsilon E_{n} \cdot \nabla p d x \quad \text { for } p \in H^{1}(D) .
$$

This implies, by 4.14, for $m, n \in \mathbb{N}$,

$$
\begin{aligned}
\int_{D} \varepsilon & \nabla\left(q_{n}-q_{m}\right) \cdot \nabla\left(\bar{q}_{n}-\bar{q}_{m}\right) d x \\
& =\int_{D} \varepsilon\left(E_{n}-E_{m}\right) \cdot \nabla\left(\bar{q}_{n}-\bar{q}_{m}\right) d x \\
& =i \int_{D}\left(\nabla \times\left(H_{n}-H_{m}\right)-\left(\theta_{2, n}-\theta_{2, m}\right)\right) \cdot \nabla\left(\bar{q}_{n}-\bar{q}_{m}\right) d x .
\end{aligned}
$$

An integration by parts yields

$$
\begin{aligned}
& \int_{D} \varepsilon \nabla\left(q_{n}-q_{m}\right) \cdot \nabla\left(\bar{q}_{n}-\bar{q}_{m}\right) d x \\
& \quad=i \int_{\partial D} \nabla \times\left(H_{n}-H_{m}\right) \cdot \nu\left(\bar{q}_{n}-\bar{q}_{m}\right) d s-i \int_{D}\left(\theta_{2, n}-\theta_{2, m}\right) \cdot \nabla\left(\bar{q}_{n}-\bar{q}_{m}\right) d x .
\end{aligned}
$$


By 4.15 and the convergence of $\left(\theta_{1, n}, \theta_{2, n}\right)$ in $\left[L^{2}(D)\right]^{6}$, the left-hand side of the above identity converges to 0 as $m, n \rightarrow \infty$. Hence, by the ellipticity of $\varepsilon,\left(\nabla q_{n}\right)_{n}$ is a Cauchy sequence and thus converges in $\left[L^{2}(D)\right]^{3}$. From 4.16 , we have

$$
\int_{D} \varepsilon^{-1} \nabla \times\left(\phi_{n}-\phi_{m}\right) \cdot \nabla \times\left(\bar{\phi}_{n}-\bar{\phi}_{m}\right) d x=\int_{D} \nabla \times\left(E_{n}-E_{m}\right) \cdot\left(\bar{\phi}_{n}-\bar{\phi}_{m}\right) d x .
$$

By the ellipticity of $\varepsilon$ and the convergence of $\left(\phi_{n}\right)$ in $L^{2}(D)$, we derive that $\left(\nabla \times \phi_{n}\right)_{n}$ is a Cauchy sequence in $\left[L^{2}(D)\right]^{3}$ and thus converges in $\left[L^{2}(D)\right]^{3}$. Since

$$
E_{n}=\nabla q_{n}+\varepsilon^{-1} \nabla \times \phi_{n}
$$

$\left(E_{n}\right)_{n}$ converges in $\left[L^{2}(D)\right]^{3}$.

Similarly, up to a subsequence, $\left(H_{n}\right)_{n}$ converges in $\left[L^{2}(D)\right]^{3}$.

Using Lemma 4.3 and applying the Fredhom theory, one can prove the wellposedness of $\left(E_{0}, H_{0}\right)$ in Definitions 2.3 and 2.4. The first result in this direction is as follows.

Lemma 4.4. Let $\theta_{1}, \theta_{2} \in\left[L^{2}(D)\right]^{3}$. The system

$$
\begin{cases}\nabla \times E=i \mu H+\theta_{1} & \text { in } D, \\ \nabla \times H=-i \varepsilon E+\theta_{2} & \text { in } D, \\ \nabla \times E \cdot \nu=\nabla \times H \cdot \nu=0 & \text { on } \partial D\end{cases}
$$

has a solution $(E, H)$ in $[H(\operatorname{curl}, D)]^{2}$ if and only if

$$
\int_{D} \theta_{2} \cdot \overline{\boldsymbol{E}} d x-\int_{D} \theta_{1} \cdot \overline{\boldsymbol{H}} d x=0 \quad \text { for all }(\boldsymbol{E}, \boldsymbol{H}) \in \mathcal{N}(D) .
$$

In particular, system 4.18 has a unique solution $(E, H) \in \mathcal{N}(D)^{\perp}$ if and only if 4.19 holds.

Proof. Lemma 4.4 is derived from the Fredholm theory. Since $\varepsilon$ and $\mu$ are uniformly elliptic, by Lemma 3.3 there exist $p_{1}, p_{2} \in H^{1}(D)$ and $\phi_{1}, \phi_{2} \in\left[H^{1}(D)\right]^{3}$ such that

$$
\theta_{1}=\mu \nabla p_{1}+\nabla \times \phi_{1}, \quad \theta_{2}=\varepsilon \nabla p_{2}+\nabla \times \phi_{2} \quad \text { in } D
$$

and

$$
\nabla \times \phi_{1} \cdot \nu=\nabla \times \phi_{2} \cdot \nu=0 \text { on } \partial D .
$$

Set $\left(E_{0}, H_{0}\right):=\left(-i \nabla p_{2}, i \nabla p_{1}\right)$ in $D$. Then $\left(E_{0}, H_{0}\right) \in[H(\operatorname{curl}, D)]^{2}$ is a solution to

$$
\begin{cases}\nabla \times E_{0}=i \mu H_{0}+\mu \nabla p_{1} & \text { in } D, \\ \nabla \times H_{0}=-i \varepsilon E_{0}+\varepsilon \nabla p_{2} & \text { in } D, \\ \nabla \times E_{0} \cdot \nu=\nabla \times H_{0} \cdot \nu=0 & \text { on } \partial D .\end{cases}
$$

We have

$$
\int_{D} \varepsilon \nabla p_{2} \cdot \overline{\mathbf{E}} d x-\int_{D} \mu \nabla p_{1} \cdot \overline{\mathbf{H}} d x=0 \quad \text { for all }(\mathbf{E}, \mathbf{H}) \in \mathcal{N}(D) .
$$


From 4.20 - 4.23), by considering $\left(E-E_{0}, H-H_{0}\right)$ instead of $(E, H)$, one might assume that $\left(\theta_{1}, \theta_{2}\right) \in H(\operatorname{div}, D)$,

$$
\operatorname{div}\left(\theta_{1}\right)=\operatorname{div}\left(\theta_{2}\right)=0 \quad \text { in } D \quad \text { and } \quad \theta_{1} \cdot \nu=\theta_{2} \cdot \nu=0 \quad \text { on } \partial D \text {. }
$$

This is assumed from now on.

Set

$$
\mathbb{V}=\{\varphi \in H(\operatorname{curl}, D): \operatorname{div}(\varepsilon \varphi)=0, \varepsilon \varphi \cdot \nu=0 \text { on } \partial D, \nabla \times \varphi \cdot \nu=0 \text { on } \partial D\} .
$$

Since $\varepsilon$ and $\mu$ are real, symmetric and uniformly elliptic, $\mathbb{V}$ is a Hilbert space equipped with the scalar product

$$
\langle E, \varphi\rangle_{\mathbb{V}, \mathbb{V}}=\int_{D} \mu^{-1} \nabla \times E \cdot \nabla \times \bar{\varphi} d x+\int_{D} \varepsilon E \cdot \bar{\varphi} d x \quad \text { for } E, \varphi \in \mathbb{V} .
$$

Let $A: \mathbb{V} \rightarrow \mathbb{V}$ be defined by

$$
\langle A E, \varphi\rangle_{\langle\mathbb{V}, \mathbb{V}\rangle}=-2 \int_{D} \varepsilon E \cdot \bar{\varphi} d x \quad \text { for all } \varphi \in \mathbb{V} .
$$

Since $\varepsilon$ is symmetric, one can easily check that $A$ is self-adjoint. Since $\varepsilon$ and $\mu$ are symmetric and uniformly elliptic, by Lemma 3.7, $A$ is compact.

Let $g \in \mathbb{V}$ be such that

$$
\langle g, \varphi\rangle_{\langle\mathbb{V}, \mathbb{V}\rangle}=\int_{D} i \theta_{2} \cdot \bar{\varphi}+\int_{D} \mu^{-1} \theta_{1} \cdot \nabla \times \bar{\varphi} \quad \text { for all } \varphi \in \mathbb{V} .
$$

We claim that

system 4.18 has a solution in $[H(\operatorname{curl}, D)]^{2}$

if and only if the equation $u+A u=g$ in $\mathbb{V}$ has a solution in $\mathbb{V}$

and

$$
\begin{aligned}
& (E, H) \text { is a solution of } 4.18 \text { if and only if } \\
& \qquad E+A E=g \text { in } \mathbb{V} \text { and } H=-i \mu^{-1}\left(\nabla \times E-\theta_{1}\right) .
\end{aligned}
$$

Assuming this, we continue the proof. By 4.28 and the Fredholm theory, see e.g. Chap. 6 of Ref. 8, system 4.18 has a solution if and only if

$$
\langle g, \varphi\rangle_{\mathbb{V}, \mathbb{V}}=0 \quad \text { for all } \varphi \in \mathbb{V} \quad \text { such that } \varphi+A \varphi=0 \quad \text { in } \mathbb{V}
$$

since $A$ is self-adjoint. Applying 4.29 with $g=\theta_{1}=\theta_{2}=0$ and using 4.25 (4.27), we derive that condition 4.30 is equivalent to the fact that

$$
\int_{D} \theta_{2} \cdot \overline{\mathbf{E}} d x-\int_{D} \theta_{1} \cdot \overline{\mathbf{H}} d x=0 \quad \text { for all }(\mathbf{E}, \mathbf{H}) \in \mathcal{N}(D),
$$

which is 4.19.

The rest of the proof is devoted to establishing Claims 4.28 and 4.29). Let $(E, H) \in[H(\operatorname{curl}, D)]^{2}$ be a solution to 4.18. From 4.24 , we derive that $E \in \mathbb{V}$. 
Fix $\varphi \in \mathbb{V}$. Then $\nabla \times \varphi \cdot \nu=0$ on $\partial D$. By Lemma 3.2 there exists $\varphi_{0} \in\left[H^{1}(D)\right]^{3}$ such that

$$
\nabla \times \varphi_{0}=\nabla \times \varphi \quad \text { in } D, \quad \operatorname{div} \varphi_{0}=0 \quad \text { in } D, \quad \text { and } \quad \varphi_{0} \times \nu=0 \quad \text { on } \partial D .
$$

Since $\nabla \times\left(\varphi_{0}-\varphi\right)=0$ and $D$ is simply connected, there exists $\xi \in H^{1}(D)$ such that

$$
\varphi_{0}-\varphi=\nabla \xi \quad \text { in } D
$$

We have, for $\varphi \in \mathbb{V}$,

$$
\int_{D} \mu^{-1} \nabla \times E \cdot \nabla \times \bar{\varphi} d x=i \int_{D} H \cdot \nabla \times \bar{\varphi}+\mu^{-1} \theta_{1} \cdot \nabla \times \bar{\varphi} d x .
$$

Using 4.31 and an integration by parts, we obtain

$$
\int_{D} H \cdot \nabla \times \bar{\varphi} d x=\int_{D} H \cdot \nabla \times \bar{\varphi}_{0} d x=\int_{D} \nabla \times H \cdot \bar{\varphi}_{0} d x .
$$

Using 4.32 and the fact $\nabla \times H \cdot \nu=0$ on $\partial D$, we also get, by an integration by parts,

$$
\int_{D} \nabla \times H \cdot \bar{\varphi}_{0} d x=\int_{D} \nabla \times H \cdot \bar{\varphi} d x
$$

This implies, by 4.34,

$$
\int_{D} H \cdot \nabla \times \bar{\varphi} d x=\int_{D} \nabla \times H \cdot \bar{\varphi} d x .
$$

A combination of 4.33 and 4.35 yields

$$
\int_{D} \mu^{-1} \nabla \times E \cdot \nabla \times \bar{\varphi} d x=i \int_{D} \nabla \times H \cdot \bar{\varphi}+\mu^{-1} \theta_{1} \cdot \nabla \times \bar{\varphi} d x .
$$

We derive from 4.18 and 4.36 that

$$
\int_{D} \mu^{-1} \nabla \times E \cdot \nabla \times \bar{\varphi} d x=\int_{D} \varepsilon E \cdot \bar{\varphi} d x+i \int_{D} \theta_{2} \cdot \bar{\varphi} d x+\int_{D} \mu^{-1} \theta_{1} \cdot \nabla \times \bar{\varphi} d x .
$$

It follows from 4.25- 4.27 that

$$
E+A E=g \text { in } \mathbb{V} .
$$

Conversely, assume that there exists $u \in \mathbb{V}$ such that $u+A u=g$. Set

$$
E=u \quad \text { and } \quad H=-i \mu^{-1}\left(\nabla \times E-\theta_{1}\right) \text { in } D .
$$

Using 4.37), one can check that $(E, H)$ satisfies the first two equations of 4.18). It is clear that $\nabla \times E \cdot \nu=0$ on $\partial D$ by the definition of $\mathbb{V}$. Since $\nabla \times H=-i \varepsilon E+\theta_{2}$ in $D, \varepsilon E \cdot \nu=0$ on $\partial D(E \in \mathbb{V})$, and $\theta_{2} \cdot \nu=0$ on $\partial D$ by 4.24, we obtain

$$
\nabla \times H \cdot \nu=0 \text { on } \partial D \text {. }
$$

The proof is complete. 
Remark 4.3. One of the key points in the proof of Lemma 4.4 is the identity

$$
\int_{D} H \cdot \nabla \times \bar{E} d x=\int_{D} \nabla \times H \cdot \bar{E} d x
$$

if $E, H \in H(\operatorname{curl}, D)$ is such that $\nabla \times E \cdot \nu=\nabla \times H \cdot \nu=0$ on $\partial D$, see 4.35). This ensures the variational character of system 4.18.

The following lemma yields the uniqueness of $\left(E_{0}, H_{0}\right)$ in Definition 2.4.

Lemma 4.5. Let $[(E, H),(\widetilde{E}, \widetilde{H})] \in\left[H_{\mathrm{loc}}\left(\operatorname{curl}, \mathbb{R}^{3}\right)\right]^{2} \times \mathcal{N}(D)^{\perp}$ be such that

$$
\left\{\begin{array} { l l } 
{ \nabla \times E = \nabla \times H = 0 } & { \text { in } \mathbb { R } ^ { 3 } \backslash D , } \\
{ \operatorname { d i v } E = \operatorname { d i v } H = 0 } & { \text { in } \mathbb { R } ^ { 3 } \backslash D , } \\
{ \nabla \times E = i \mu H } & { \text { in } D , } \\
{ \nabla \times H = - i \varepsilon E } & { \text { in } D , }
\end{array} \quad \text { and } \quad \left\{\begin{array}{ll}
\nabla \times \widetilde{E}=i \mu \widetilde{H} & \text { in } D, \\
\nabla \times \widetilde{H}=-i \varepsilon \widetilde{E} & \text { in } D, \\
\varepsilon \widetilde{E} \cdot \nu=\left.E \cdot \nu\right|_{\text {ext }} & \text { on } \partial D, \\
\mu \widetilde{H} \cdot \nu=\left.H \cdot \nu\right|_{\text {ext }} & \text { on } \partial D,
\end{array}\right.\right.
$$

and

$$
|(E(x), H(x))|=O\left(|x|^{-2}\right) \quad \text { for large }|x| .
$$

Then $(E, H)=(0,0)$ in $\mathbb{R}^{3}$ and $(\widetilde{E}, \widetilde{H})=(0,0)$ in $D$.

Proof. Applying Lemma 3.1 to $\bar{E}$, there exists a function $\theta \in H_{\text {loc }}^{1}\left(\mathbb{R}^{3} \backslash D\right)$ such that

$$
\nabla \theta=\bar{E} \quad \text { in } \mathbb{R}^{3} \backslash D \quad \text { and } \quad|\theta(x)|=O\left(|x|^{-1}\right) \quad \text { for large }|x| .
$$

For $R>0$ large, since $\operatorname{div} E=0$ in $\mathbb{R}^{3} \backslash D$, we have

$$
\int_{B_{R} \backslash D}|E|^{2} d x=\int_{B_{R} \backslash D} E \cdot \nabla \theta d x=\int_{\partial B_{R}}(E \cdot \nu) \theta d s-\left.\int_{\partial D}(E \cdot \nu)\right|_{\text {ext }} \theta d s .
$$

Letting $R$ tend to $+\infty$ and using 4.39) and 4.40, we obtain

$$
\int_{\mathbb{R}^{3} \backslash D}|E|^{2} d x=-\left.\int_{\partial D}(E \cdot \nu)\right|_{\mathrm{ext}} \theta d s .
$$

Extend $\theta$ in $D$ so that the extension belongs to $H_{\text {loc }}^{1}\left(\mathbb{R}^{3}\right)$ and still denote this extension by $\theta$. We derive from the system of $(\widetilde{E}, \widetilde{H})$ in 4.38 that

$$
\begin{aligned}
-\left.\int_{\partial D}(E \cdot \nu)\right|_{\text {ext }} \theta d s & =-\int_{\partial D}(\varepsilon \widetilde{E} \cdot \nu) \theta d s=-\int_{D} \varepsilon \widetilde{E} \cdot \nabla \theta d x-\int_{D} \operatorname{div}(\varepsilon \widetilde{E}) \theta d x \\
& =\int_{D}-i \nabla \times \widetilde{H} \cdot \nabla \theta d x=-i \int_{\partial D} \widetilde{H} \cdot(\nabla \theta \times \nu) d s \\
& =-i \int_{\partial D} \widetilde{H} \cdot(\bar{E} \times \nu) d s
\end{aligned}
$$


Combining 4.41 and 4.42 yields

$$
\int_{\mathbb{R}^{3} \backslash D}|E|^{2} d x=-i \int_{\partial D} \widetilde{H} \cdot(\bar{E} \times \nu) d s .
$$

Similarly, we have

$$
\int_{\mathbb{R}^{3} \backslash D}|H|^{2} d x=i \int_{\partial D} \widetilde{E} \cdot(\bar{H} \times \nu) d s .
$$

An integration by parts implies

$$
\begin{aligned}
\int_{\partial D} & \widetilde{H} \cdot(\bar{E} \times \nu) d s-\int_{\partial D} \widetilde{E} \cdot(\bar{H} \times \nu) d s \\
\quad & =\int_{D} \nabla \times \widetilde{H} \cdot \bar{E} d x-\int_{D} \nabla \times \bar{E} \cdot \widetilde{H} d x-\int_{D} \nabla \times \widetilde{E} \cdot \bar{H} d x+\int_{D} \nabla \times \bar{H} \cdot \widetilde{E} d x .
\end{aligned}
$$

Using the equations of $(E, H)$ and $(\widetilde{E}, \widetilde{H})$ in $D$ in 4.38), we obtain

$$
\int_{\partial D} \widetilde{H} \cdot(\bar{E} \times \nu) d s-\int_{\partial D} \widetilde{E} \cdot(\bar{H} \times \nu) d s=0 .
$$

A combination of 4.43-4.45 yields

$$
\int_{\mathbb{R}^{3} \backslash D}\left(|E|^{2}+|H|^{2}\right) d x=0 .
$$

We derive that $E=H=0$ in $\mathbb{R}^{3} \backslash D$. This implies, by the unique continuation principle, see e.g. Theorem 1 of Ref. 33 ,

$$
E=H=0 \text { in } D
$$

and, since $(\widetilde{E}, \widetilde{H}) \in \mathcal{N}(D)^{\perp}$,

$$
\widetilde{E}=\widetilde{H}=0 \quad \text { in } D \text {. }
$$

The proof is complete.

\subsection{Approximate cloaking in the non-resonant case - Proof of Theorem 2.1}

The key ingredient in the proof of Theorem 2.1 is the following lemma whose proof uses various results in Secs. 3 and 4.1.

Lemma 4.6. Let $0<\rho<\rho_{0}, \theta_{\rho}=\left(\theta_{1, \rho}, \theta_{2, \rho}\right) \in\left[L^{2}(D)\right]^{6}$, and $h_{\rho}=\left(h_{1, \rho}, h_{2, \rho}\right) \in$ $\left[H^{-1 / 2}\left(\operatorname{div}_{\Gamma}, \partial D\right)\right]^{2}$. Let $\left(E_{\rho}, H_{\rho}\right) \in\left[\bigcap_{R>1} H\left(\operatorname{curl}, B_{R} \backslash \partial D\right)\right]^{2}$ be the unique radiating solution to the system

$$
\begin{cases}\nabla \times E_{\rho}=i \rho H_{\rho} & \text { in } \mathbb{R}^{3} \backslash D, \\ \nabla \times H_{\rho}=-i \rho E_{\rho} & \text { in } \mathbb{R}^{3} \backslash D, \\ \nabla \times E_{\rho}=i \mu H_{\rho}+\theta_{1, \rho} & \text { in } D, \\ \nabla \times H_{\rho}=-i \varepsilon E_{\rho}+\theta_{2, \rho} & \text { in } D, \\ {\left[E_{\rho} \times \nu\right]=h_{1, \rho},\left[H_{\rho} \times \nu\right]=h_{2, \rho}} & \text { on } \partial D .\end{cases}
$$


Assume that $\mathcal{N}(D)=\{(0,0)\}$. We have

$$
\left\|\left(E_{\rho}, H_{\rho}\right)\right\|_{L^{2}\left(B_{5}\right)} \leq C\left(\left\|\theta_{\rho}\right\|_{L^{2}(D)}+\left\|h_{\rho}\right\|_{H^{-1 / 2}\left(\operatorname{div}_{\Gamma}, \partial D\right)}\right)
$$

for some positive constant $C$ depending only on $\rho_{0}, \varepsilon, \mu$. Assume in addition that

$$
\lim _{\rho \rightarrow 0}\left\|h_{\rho}\right\|_{H^{-1 / 2}\left(\operatorname{div}_{\Gamma}, \partial D\right)}=0 \quad \text { and } \quad \lim _{\rho \rightarrow 0} \theta_{\rho}=\theta \quad \text { in }\left[L^{2}(D)\right]^{6}
$$

for some $\theta=\left(\theta_{1}, \theta_{2}\right) \in\left[L^{2}(D)\right]^{6}$. We have

$$
\lim _{\rho \rightarrow 0}\left(E_{\rho}, H_{\rho}\right)=C l\left(\theta_{1}, \theta_{2}\right) \quad \text { in }[H(\operatorname{curl}, D)]^{2} .
$$

Here and in what follows on $\partial D,[u]$ denotes the jump of $u$ across $\partial D$ for an appropriate (vectorial) function $u$, i.e. $[u]=\left.u\right|_{\text {ext }}-\left.u\right|_{\text {int }}$ on $\partial D$.

Proof. By Lemma 3.8, without loss of generality, one might assume that $h_{1, \rho}=$ $h_{2, \rho}=0$ on $\partial D$. This is assumed from now on.

We first prove 4.46 by contradiction. Assume that there exist sequences $\left(\rho_{n}\right)_{n} \subset\left(0, \rho_{0}\right),\left(\left(E_{n}, H_{n}\right)\right)_{n} \subset\left[H_{\mathrm{loc}}\left(\operatorname{curl}, \mathbb{R}^{3}\right)\right]^{2},\left(\left(\theta_{1, n}, \theta_{2, n}\right)\right)_{n} \subset\left[L^{2}(D)\right]^{6}$ such that

$$
\begin{aligned}
& \begin{cases}\nabla \times E_{n}=i \rho_{n} H_{n} & \text { in } \mathbb{R}^{3} \backslash D, \\
\nabla \times H_{n}=-i \rho_{n} E_{n} & \text { in } \mathbb{R}^{3} \backslash D, \\
\nabla \times E_{n}=i \mu H_{n}+\theta_{1, n} & \text { in } D, \\
\nabla \times H_{n}=-i \varepsilon E_{n}+\theta_{2, n} & \text { in } D,\end{cases} \\
& \left\|\left(E_{n}, H_{n}\right)\right\|_{L^{2}\left(B_{5}\right)}=1 \quad \text { for all } n \in \mathbb{N},
\end{aligned}
$$

and

$$
\lim _{n \rightarrow+\infty}\left\|\left(\theta_{1, n}, \theta_{2, n}\right)\right\|_{L^{2}(D)}=0 .
$$

Without loss of generality, one might assume that $\rho_{n} \rightarrow \rho_{\infty} \in\left[0, \rho_{0}\right]$. We only consider the case $\rho_{\infty}=0$. The case $\rho_{\infty}>0$ can be proved similarly.

We have

$$
\nabla \times\left. E_{n} \cdot \nu\right|_{\mathrm{int}}=\nabla \times\left. E_{n} \cdot \nu\right|_{\mathrm{ext}}=\left.i \rho_{n} H_{n} \cdot \nu\right|_{\mathrm{ext}} \rightarrow 0 \quad \text { in } H^{-1 / 2}(\partial D) \text { as } n \rightarrow \infty \text {. }
$$

Similarly, we obtain

$$
\nabla \times\left. H_{n} \cdot \nu\right|_{\text {int }} \rightarrow 0 \text { in } H^{-1 / 2}(\partial D) \text { as } n \rightarrow \infty .
$$

Applying Lemma 4.3 to $\left(\left(E_{n}, H_{n}\right)\right)_{n}$ in $D$, without loss of generality, one might assume that

$$
\left(\left(E_{n}, H_{n}\right)\right)_{n} \text { converges in }[H(\operatorname{curl}, D)]^{2} \text { as } n \rightarrow \infty \text {. }
$$

Applying (i) of Lemma 3.8, we derive that

$$
\left(\left(E_{n} \times \nu, H_{n} \times \nu\right)\right)_{n} \text { converges in }\left[H^{-1 / 2}\left(\operatorname{div}_{\Gamma}, \partial D\right)\right]^{2} \text { as } n \rightarrow \infty \text {. }
$$


It follows from 4.48, Lemma 3.6, and (i) of Lemma 3.7 that

$$
\left(\left(E_{n}, H_{n}\right)\right)_{n} \text { converges in }\left[L_{\text {loc }}^{2}\left(\mathbb{R}^{3} \backslash D\right)\right]^{6} \text { as } n \rightarrow \infty .
$$

Let $(E, H)$ be the limit of $\left(E_{n}, H_{n}\right)$ in $\left[L_{\text {loc }}^{2}\left(\mathbb{R}^{3}\right)\right]^{6}$. Then $(E, H) \in$ $\left[H_{\text {loc }}\left(\operatorname{curl}, \mathbb{R}^{3}\right)\right]^{2}$ and $^{\mathrm{b}}$

$$
\begin{cases}\nabla \times E=\nabla \times H=0 & \text { in } \mathbb{R}^{3} \backslash D, \\ \operatorname{div} E=\operatorname{div} H=0 & \text { in } \mathbb{R}^{3} \backslash D, \\ \nabla \times E=i \mu H & \text { in } D, \\ \nabla \times H=-i \varepsilon E & \text { in } D .\end{cases}
$$

We derive from 4.49 and 4.50 that

$$
\nabla \times\left. E \cdot \nu\right|_{\text {int }}=\nabla \times\left. H \cdot \nu\right|_{\text {int }}=0 \quad \text { on } \partial D .
$$

Applying Lemma 3.6, we have

$$
|(E(x), H(x))| \leq \frac{C}{|x|^{2}} \quad \text { for }|x|>3
$$

for some positive constant $C$. Combining (4.53) and (4.54) yields that $\left.(E, H)\right|_{D} \in$ $\mathcal{N}(D)$. Since $\mathcal{N}(D)=\{(0,0)\}$, it follows that $E=H=0$ in $D$. Hence

$$
E \times \nu=H \times \nu=0 \quad \text { on } \partial D .
$$

We have, for each connected component $\Gamma$ of $\partial D$,

$$
\left.\int_{\Gamma} E \cdot \nu\right|_{\mathrm{ext}}=\left.\lim _{n \rightarrow \infty} \int_{\Gamma} E_{n}\right|_{\mathrm{ext}} \cdot \nu=\left.\lim _{n \rightarrow \infty} \frac{1}{-i \rho_{n}} \int_{\Gamma}\left(\nabla \times H_{n}\right) \cdot \nu\right|_{\mathrm{ext}}=0
$$

and similarly,

$$
\left.\int_{\Gamma} H \cdot \nu\right|_{\text {ext }}=0
$$

Using 4.53, 4.55, and 4.56, and applying Lemma 3.5 to $(E, H)$ in $\mathbb{R}^{3} \backslash D$, we obtain

$$
E=H=0 \quad \text { in } \mathbb{R}^{3} \backslash D .
$$

Thus $E=H=0$ in $\mathbb{R}^{3}$, which, by using 4.51) and 4.52, contradicts 4.48). Therefore, 4.46 is proved.

We next establish 4.47). Fix an arbitrary sequence $\left(\rho_{n}\right)_{n}$ converging to 0. From (4.46), one obtains that

$$
\left\|\left(E_{\rho_{n}}, H_{\rho_{n}}\right)\right\|_{L^{2}\left(B_{5}\right)} \leq C\left(\left\|\theta_{\rho_{n}}\right\|_{L^{2}(D)}+\left\|h_{\rho_{n}}\right\|_{H^{-1 / 2}\left(\operatorname{div}_{\Gamma}, \partial D\right)}\right) \leq C .
$$

\footnotetext{
${ }^{\mathrm{b}}$ In the case $\rho_{\infty}>0$, the limit $(E, H)$ satisfies the radiating condition and is a solution to Maxwell equations in $\mathbb{R}^{3}$ with vanished data. It follows that $(E, H)=(0,0)$, which also gives a contradiction.
} 
Using the same argument as above, one obtains that, up to a subsequence, $\left(E_{\rho_{n}}, H_{\rho_{n}}\right)$ converges in $\left[H\left(\operatorname{curl}, \mathbb{R}^{3}\right)\right]^{2}$ to $(E, H)$, the unique solution of

$$
\begin{cases}\nabla \times E=\nabla \times H=0 & \text { in } \mathbb{R}^{3} \backslash D, \\ \operatorname{div} E=\operatorname{div} H=0 & \text { in } \mathbb{R}^{3} \backslash D, \\ \nabla \times E=i \mu H+\theta_{1} & \text { in } D, \\ \nabla \times H=-i \varepsilon E+\theta_{2} & \text { in } D .\end{cases}
$$

This system implies $\nabla \times\left. E \cdot \nu\right|_{\text {int }}=\nabla \times\left. H \cdot \nu\right|_{\text {int }}=0$ on $\partial D$. Since $\mathcal{N}(D)=\{(0,0)\}$, we have $\left.(E, H)\right|_{D}=C l\left(\theta_{1}, \theta_{2}\right)$. Since $\left(\rho_{n}\right) \rightarrow 0$ arbitrarily, assertion 4.47 follows. The proof is complete.

We are ready to give the following.

Proof of Theorem 2.1. Let $\left(E_{1, \rho}, H_{1, \rho}\right) \in\left[H_{\text {loc }}\left(\operatorname{curl}, \mathbb{R}^{3} \backslash B_{\rho}\right)\right]^{2}$ be the unique radiating solution to the system

$$
\begin{cases}\nabla \times E_{1, \rho}=i \omega H_{1, \rho} & \text { in } \mathbb{R}^{3} \backslash B_{\rho} \\ \nabla \times H_{1, \rho}=-i \omega E_{1, \rho}+J_{\text {ext }} & \text { in } \mathbb{R}^{3} \backslash B_{\rho} \\ E_{1, \rho} \times \nu=0 & \text { on } \partial B_{\rho}\end{cases}
$$

extend $\left(E_{1, \rho}, H_{1, \rho}\right)$ by $(0,0)$ in $B_{\rho}$, and still denote this extension by $\left(E_{1, \rho}, H_{1, \rho}\right)$. Define, in $\mathbb{R}^{3}$,

$\left(E_{2, \rho}, H_{2, \rho}\right):=(E, H)-\left(E_{1, \rho}, H_{1, \rho}\right) \quad$ and $\quad\left(E_{3, \rho}, H_{3, \rho}\right):=\left(\mathcal{E}_{\rho}, \mathcal{H}_{\rho}\right)-\left(E_{1, \rho}, H_{1, \rho}\right)$. Then $\left(E_{2, \rho}, H_{2, \rho}\right) \in\left[H_{\text {loc }}\left(\operatorname{curl}, \mathbb{R}^{3} \backslash B_{\rho}\right)\right]^{2}$ is the unique radiating solution to the system

$$
\begin{cases}\nabla \times E_{2, \rho}=i \omega H_{2, \rho} & \text { in } \mathbb{R}^{3} \backslash B_{\rho} \\ \nabla \times H_{2, \rho}=-i \omega E_{2, \rho} & \text { in } \mathbb{R}^{3} \backslash B_{\rho} \\ E_{2, \rho} \times \nu=E \times \nu & \text { on } \partial B_{\rho}\end{cases}
$$

and $\left(E_{3, \rho}, H_{3, \rho}\right) \in\left[\bigcap_{R>1} H\left(\operatorname{curl}, B_{R} \backslash \partial B_{\rho}\right)\right]^{2}$ is the unique radiating solution to the system

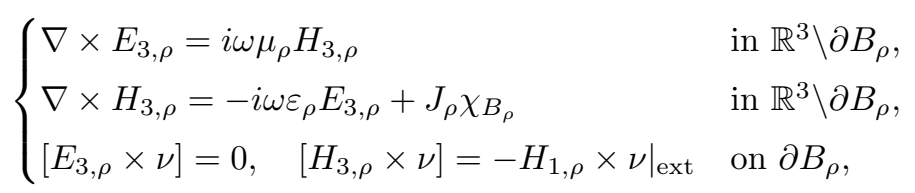

where $\chi_{D}$ denotes the characteristic function of a subset $D$ of $\mathbb{R}^{3}$. Recall that $J_{\rho}$ is defined in 2.15. Set

$$
\widetilde{E}_{2, \rho}(x)=E_{\rho}(\rho x) \quad \text { and } \quad \widetilde{H}_{2, \rho}(x)=H_{\rho}(\rho x) \quad \text { for } x \in \mathbb{R}^{3} \backslash B_{1} .
$$


Then $\left(\widetilde{E}_{2, \rho}, \widetilde{H}_{2, \rho}\right) \in\left[H\left(\operatorname{curl}, \mathbb{R}^{3} \backslash B_{1}\right)\right]^{2}$ is the unique radiating solution to the system

$$
\begin{cases}\nabla \times \widetilde{E}_{2, \rho}=i \omega \rho \widetilde{H}_{2, \rho} & \text { in } \mathbb{R}^{3} \backslash B_{1}, \\ \nabla \times \widetilde{H}_{2, \rho}=-i \omega \rho \widetilde{E}_{2, \rho} & \text { in } \mathbb{R}^{3} \backslash B_{1}, \\ \widetilde{E}_{2, \rho} \times \nu=E(\rho \cdot) \times \nu & \text { on } \partial B_{1} .\end{cases}
$$

By Lemmas 4.1 and 4.2 (also Remark 4.1), we have, for $R>1 / 2$ and for $x \in$ $B_{3 R} \backslash B_{2 R}$,

$$
\begin{aligned}
\left|\left(\widetilde{E}_{2, \rho}\left(\frac{x}{\rho}\right), \widetilde{H}_{2, \rho}\left(\frac{x}{\rho}\right)\right)\right| \leq & C \rho^{3}\left\|\left(\widetilde{E}_{2, \rho}, \widetilde{H}_{2, \rho}\right)\right\|_{L^{2}\left(B_{2} \backslash B_{1}\right)} \\
\leq & C \rho^{3}\left(\|E(\rho .) \times \nu\|_{H^{-1 / 2}\left(\partial B_{1}\right)}\right. \\
& \left.+\rho^{-1}\left\|\operatorname{div}_{\partial B_{1}}(E(\rho .) \times \nu)\right\|_{H^{-1 / 2}\left(\partial B_{1}\right)}\right) \\
\leq & C \rho^{3}\left(\|E(\rho .) \times \nu\|_{H^{-1 / 2}\left(\partial B_{1}\right)}+\|H(\rho .) \cdot \nu\|_{H^{-1 / 2}\left(\partial B_{1}\right)}\right) .
\end{aligned}
$$

Here and in what follows in this proof, $C$ denotes a positive constant depending only on $\rho_{0}, R_{0}$, and $R$. It follows from the definition of $\left(\widetilde{E}_{2, \rho}, \widetilde{H}_{2, \rho}\right)$ that

$$
\left\|\left(E_{2, \rho}, H_{2, \rho}\right)\right\|_{L^{2}\left(B_{3 R} \backslash B_{2 R}\right)} \leq C \rho^{3}\left\|J_{\text {ext }}\right\|_{L^{2}\left(\mathbb{R}^{3} \backslash B_{2}\right)} .
$$

From now on in this proof, for any vector field $v$, we denote ${ }^{c}$

$$
\hat{v}(\cdot):=\rho v(\rho \cdot) .
$$

We claim that

$$
\left\|\hat{H}_{1, \rho} \times\left.\nu\right|_{\mathrm{ext}}\right\|_{H^{-1 / 2}\left(\partial B_{1}\right)}+\left\|\left.\hat{E}_{1, \rho} \cdot \nu\right|_{\mathrm{ext}}\right\|_{H^{-1 / 2}\left(\partial B_{1}\right)} \leq C \rho\left\|J_{\mathrm{ext}}\right\|_{L^{2}\left(\mathbb{R}^{3} \backslash B_{2}\right)}
$$

and, for $R>1 / 2$,

$$
\left\|\left(E_{3, \rho}, H_{3, \rho}\right)\right\|_{L^{2}\left(B_{3 R} \backslash B_{2 R}\right)} \leq C\left(\rho^{3}\left\|J_{\text {ext }}\right\|_{L^{2}\left(\mathbb{R}^{3} \backslash B_{2}\right)}+\rho^{2}\left\|J_{\text {int }}\right\|_{L^{2}\left(B_{1}\right)}\right) .
$$

It is clear that 2.3 follows from 4.57) and 4.60). Moreover, by Lemma 4.6. assertion 2.4 now follows from 4.59) and the fact that $\left(E_{c}, H_{c}\right)=\left(\hat{E}_{3, \rho}, \hat{H}_{3, \rho}\right)$ in $B_{1}$.

It remains to establish 4.59 and 4.60). It is clear that $\left(\hat{E}_{3, \rho}, \hat{H}_{3, \rho}\right) \in$ $\left[\bigcap_{R>0} H\left(\operatorname{curl}, B_{R} \backslash \partial B_{1}\right)\right]^{2}$ is the unique radiating solution to the system

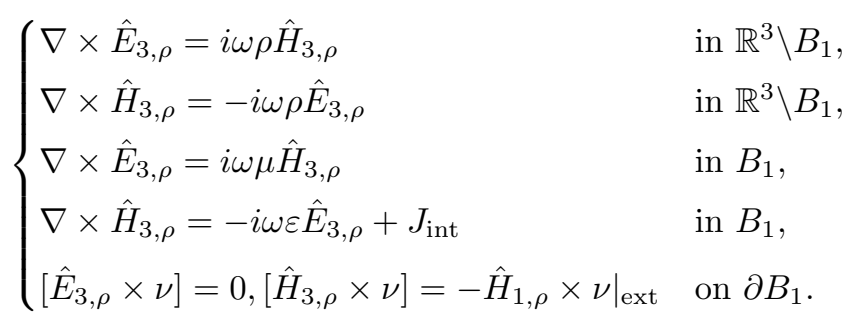

${ }^{\mathrm{c}}$ With this notation, one has $\left(E_{c}, H_{c}\right)(x)=\left(\hat{\mathcal{E}}_{\rho}, \hat{\mathcal{H}}_{\rho}\right)$ in $B_{1}$. Note that $\hat{v}(\cdot) \neq v(\rho \cdot)$. 
By Lemma 4.6, we have

$$
\left\|\left(\hat{E}_{3, \rho}, \hat{H}_{3, \rho}\right)\right\|_{H\left(\operatorname{curl}, B_{5}\right)} \leq C\left(\left\|J_{\mathrm{int}}\right\|_{L^{2}\left(B_{1}\right)}+\left\|\hat{H}_{1, \rho} \times\left.\nu\right|_{\operatorname{ext}}\right\|_{H^{-1 / 2}\left(\operatorname{div}_{\Gamma}, \partial B_{1}\right)}\right) .
$$

Applying Lemma 4.1 to $\left(\hat{E}_{2, \rho}, \hat{H}_{2, \rho}\right)$, by 4.58, we obtain

$$
\left\|\hat{H}_{2, \rho} \times\left.\nu\right|_{\text {ext }}\right\|_{H^{-1 / 2}\left(\partial B_{1}\right)}+\left\|\left.\hat{E}_{2, \rho} \cdot \nu\right|_{\text {ext }}\right\|_{H^{-1 / 2}\left(\partial B_{1}\right)} \leq C \rho\left\|J_{\text {ext }}\right\|_{L^{2}\left(\mathbb{R}^{3} \backslash B_{2}\right)} .
$$

Since

$$
\left(E_{2, \rho}, H_{2, \rho}\right)=(E, H)-\left(E_{1, \rho}, H_{1, \rho}\right) \quad \text { in } \mathbb{R}^{3} \backslash B_{1},
$$

it follows that

$$
\left\|\hat{H}_{1, \rho} \times\left.\nu\right|_{\text {ext }}\right\|_{H^{-1 / 2}\left(\partial B_{1}\right)}+\left\|\left.\hat{E}_{1, \rho} \cdot \nu\right|_{\text {ext }}\right\|_{H^{-1 / 2}\left(\partial B_{1}\right)} \leq C \rho\left\|J_{\text {ext }}\right\|_{L^{2}\left(\mathbb{R}^{3} \backslash B_{2}\right)},
$$

which is 4.59 .

Combining 4.59) and 4.61 yields

$$
\left\|\left(\hat{E}_{3, \rho}, \hat{H}_{3, \rho}\right)\right\|_{H\left(\operatorname{curl}, B_{5}\right)} \leq C\left(\left\|J_{\mathrm{int}}\right\|_{L^{2}\left(B_{1}\right)}+\rho\left\|J_{\mathrm{ext}}\right\|_{L^{2}\left(\mathbb{R}^{3} \backslash B_{2}\right)}\right) .
$$

Applying Lemma 4.2, and using 4.62, we obtain

$$
\left|\left(\hat{E}_{3, \rho}\left(\frac{x}{\rho}\right), \hat{H}_{3, \rho}\left(\frac{x}{\rho}\right)\right)\right| \leq C \rho^{3}\left(\left\|J_{\mathrm{int}}\right\|_{L^{2}\left(B_{1}\right)}+\rho\left\|J_{\mathrm{ext}}\right\|_{L^{2}\left(\mathbb{R}^{3} \backslash B_{2}\right)}\right)
$$

for $x \in B_{3 R} \backslash B_{2 R}$. This implies 4.60. The proof is complete.

\subsection{Approximate cloaking in the resonant case - Proofs of Theorems 2.2 and 2.3}

The key ingredient in the proof of Theorems 2.2 and 2.3 is the following variant of Lemma 4.6.

Lemma 4.7. Let $0<\rho<\rho_{0}, \theta_{\rho}=\left(\theta_{1, \rho}, \theta_{2, \rho}\right) \in\left[L^{2}(D)\right]^{6}$, and $h_{\rho}=\left(h_{1, \rho}, h_{2, \rho}\right) \in$ $\left[H^{-1 / 2}\left(\operatorname{div}_{\Gamma}, \partial D\right)\right]^{2}$, and let $\left(E_{\rho}, H_{\rho}\right) \in\left[\bigcap_{R>1} H\left(\operatorname{curl}, B_{R} \backslash \partial D\right)\right]^{2}$ be the unique radiating solution to the system

$$
\begin{cases}\nabla \times E_{\rho}=i \rho H_{\rho} & \text { in } \mathbb{R}^{3} \backslash D, \\ \nabla \times H_{\rho}=-i \rho E_{\rho} & \text { in } \mathbb{R}^{3} \backslash D, \\ \nabla \times E_{\rho}=i \mu H_{\rho}+\theta_{1, \rho} & \text { in } D, \\ \nabla \times H_{\rho}=-i \varepsilon E_{\rho}+\theta_{2, \rho} & \text { in } D, \\ {\left[E_{\rho} \times \nu\right]=h_{1, \rho},\left[H_{\rho} \times \nu\right]=h_{2, \rho}} & \text { on } \partial D .\end{cases}
$$

Assume that $\mathcal{N}(D) \neq\{(0,0)\}$. We have

$$
\begin{aligned}
& \left\|\left(E_{\rho}, H_{\rho}\right)\right\|_{L^{2}\left(B_{5}\right)} \\
& \quad \leq C\left(\rho^{-1}\left\|\theta_{\rho}\right\|_{L^{2}(D)}+\left\|h_{\rho}\right\|_{H^{-1 / 2}(\partial D)}+\rho^{-1}\left\|\operatorname{div}_{\Gamma} h_{\rho}\right\|_{H^{-1 / 2}(\partial D)}\right) .
\end{aligned}
$$


Assume in addition that, for all $\rho \in\left(0, \rho_{0}\right)$,

$$
\int_{D}\left(\theta_{2, \rho} \cdot \overline{\boldsymbol{E}}-\theta_{1, \rho} \cdot \overline{\boldsymbol{H}}\right) d x=0 \quad \text { for all }(\boldsymbol{E}, \boldsymbol{H}) \in \mathcal{N}(D) .
$$

Then

$$
\begin{aligned}
& \left\|\left(E_{\rho}, H_{\rho}\right)\right\|_{L^{2}\left(B_{5}\right)} \\
& \quad \leq C\left(\left\|\theta_{\rho}\right\|_{L^{2}(D)}+\left\|h_{\rho}\right\|_{H^{-1 / 2}(\partial D)}+\rho^{-1}\left\|\operatorname{div}_{\Gamma} h_{\rho}\right\|_{H^{-1 / 2}(\partial D)}\right) .
\end{aligned}
$$

Here $C$ denotes a positive constant depending only on $\rho_{0}, \varepsilon$, and $\mu$. Moreover, if $\lim _{\rho \rightarrow 0}\left(\left\|h_{\rho}\right\|_{H^{-1 / 2}(\partial D)}+\rho^{-1}\left\|\operatorname{div}_{\Gamma} h_{\rho}\right\|_{H^{-1 / 2}(\partial D)}\right)=0 \quad$ and $\quad \lim _{\rho \rightarrow 0} \theta_{\rho}=\theta$ in $\left[L^{2}(D)\right]^{6}$, for some $\theta=\left(\theta_{1}, \theta_{2}\right) \in\left[L^{2}(D)\right]^{6}$, then

$$
\lim _{\rho \rightarrow 0}\left(E_{\rho}, H_{\rho}\right)=C l\left(\theta_{1}, \theta_{2}\right) \quad \text { in }[H(\operatorname{curl}, D)]^{2} .
$$

Remark 4.4. In comparison with 4.46 in Lemma 4.6 in the resonant case $\mathcal{N}(D) \neq\{(0,0)\}$, estimate 4.63 is weaker. Under the compatibility condition 4.64, estimate 4.65 is stronger than 4.63). Note that the term $\left\|\operatorname{div}_{\Gamma} h_{\rho}\right\|_{H^{-1 / 2}(\partial D)}$ in 4.46 of Lemma 4.6 is replaced by $\rho^{-1}\left\|\operatorname{div}_{\Gamma} h_{\rho}\right\|_{H^{-1 / 2}(\partial D)}$ in 4.65. However, this does not affect the estimate for the degree of visibility in the compatible resonant case (in comparison with the non-resonant case) since in the proof of Theorem 1.2, we apply Lemma 4.7 to the situation, where $\left\|h_{\rho}\right\|_{H^{-1 / 2}(\partial D)}$ and $\rho^{-1}\left\|\operatorname{div}_{\Gamma} h_{\rho}\right\|_{H^{-1 / 2}(\partial D)}$ are of the same order. The estimates in Lemma 4.7 are somehow sharp because of the optimality of the estimates in Theorems 2.2 and 2.3 . this is discussed in Sec. 5 .

Proof. We will give the proof of 4.65 and 4.66 and explain how to modify the proof of 4.65 to obtain 4.63.

We prove 4.65) by contradiction. Assume that there exist sequences $\left(\rho_{n}\right)_{n} \subset$ $\left(0, \rho_{0}\right),\left(\left(E_{n}, H_{n}\right)\right)_{n} \subset\left[\bigcap_{R>0} H\left(\operatorname{curl}, B_{R} \backslash \partial D\right)\right]^{2},\left(\theta_{n}\right)_{n}=\left(\left(\theta_{1, n}, \theta_{2, n}\right)\right)_{n} \subset\left[L^{2}(D)\right]^{6}$ such that 4.64 holds for $\left(\theta_{1, n}, \theta_{2, n}\right)$,

$$
\begin{aligned}
& \begin{cases}\nabla \times E_{n}=i \rho_{n} H_{n} & \text { in } \mathbb{R}^{3} \backslash D, \\
\nabla \times H_{n}=-i \rho_{n} E_{n} & \text { in } \mathbb{R}^{3} \backslash D, \\
\nabla \times E_{n}=i \mu H_{n}+\theta_{1, n} & \text { in } D, \\
\nabla \times H_{n}=-i \varepsilon E_{n}+\theta_{2, n} & \text { in } D, \\
{\left[E_{n} \times \nu\right]=h_{1, n},\left[H_{n} \times \nu\right]=h_{2, n}} & \text { on } \partial D,\end{cases} \\
& \left\|\left(E_{n}, H_{n}\right)\right\|_{L^{2}\left(B_{5}\right)}=1 \quad \text { for all } n \in \mathbb{N},
\end{aligned}
$$

and

$$
\lim _{n \rightarrow+\infty}\left(\left\|\theta_{n}\right\|_{L^{2}(D)}+\left\|h_{n}\right\|_{H^{-1 / 2}(\partial D)}+\rho_{n}^{-1}\left\|\operatorname{div}_{\Gamma} h_{n}\right\|_{H^{-1 / 2}(\partial D)}\right)=0 .
$$


Without loss of generality, we assume that $\rho_{n} \rightarrow \rho_{\infty} \in\left[0, \rho_{0}\right]$. We will only consider the case $\rho_{\infty}=0$. The proof in the case $\rho_{\infty}>0$ follows similarly and is omitted.

Similar to 4.49 and 4.50, we have, by 4.68,

$$
\lim _{n \rightarrow+\infty} \nabla \times\left. E_{n}\right|_{\text {int }} \cdot \nu=0 \quad \text { and } \quad \lim _{n \rightarrow+\infty} \nabla \times\left. H_{n} \cdot \nu\right|_{\text {int }}=0 \quad \text { in } H^{-1 / 2}(\partial D) \text {. }
$$

Applying Lemma 4.3 and using (4.67), without loss of generality, one might assume that $\left(\left(E_{n}, H_{n}\right)\right)_{n}$ converges in $\left[L^{2}(D)\right]^{6}$ and hence also in $\left[L_{\text {loc }}^{2}\left(\mathbb{R}^{3} \backslash D\right)\right]^{6}$ by applying (4.3) of Lemma 4.1 and (i) of Lemma 3.7 to $B_{R} \backslash D$. Moreover, the limit $(E, H) \in$ $\left[H_{\text {loc }}\left(\text { curl, } \mathbb{R}^{3}\right)\right]^{2}$ satisfies

$$
\begin{cases}\nabla \times E=\nabla \times H=0 & \text { in } \mathbb{R}^{3} \backslash D, \\ \operatorname{div} E=\operatorname{div} H=0 & \text { in } \mathbb{R}^{3} \backslash D, \\ \nabla \times E=i \mu H & \text { in } D, \\ \nabla \times H=-i \varepsilon E & \text { in } D,\end{cases}
$$

and, by applying Lemma 3.6 and letting $\rho_{n} \rightarrow 0$,

$$
|(E(x), H(x))|=O\left(|x|^{-2}\right) \quad \text { for large }|x| .
$$

Since

$$
\int_{D}\left(\theta_{2, n} \cdot \overline{\mathbf{E}}-\theta_{1, n} \cdot \overline{\mathbf{H}}\right) d x=0 \quad \text { for all }(\mathbf{E}, \mathbf{H}) \in \mathcal{N}(D),
$$

by Lemma 4.4 there exists a unique $\left(E_{1, n}, H_{1, n}\right) \in \mathcal{N}(D)^{\perp}$ solving

$$
\begin{cases}\nabla \times E_{1, n}=i \mu H_{1, n}+\theta_{1, n} & \text { in } D, \\ \nabla \times H_{1, n}=-i \varepsilon E_{1, n}+\theta_{2, n} & \text { in } D, \\ \nabla \times E_{1, n} \cdot \nu=\nabla \times H_{1, n} \cdot \nu=0 & \text { on } \partial D .\end{cases}
$$

Denote by $\left(E_{2, n}, H_{2, n}\right)$ the projection of $\left(E_{n}, H_{n}\right)-\left(E_{1, n}, H_{1, n}\right)$ onto $\mathcal{N}(D)$ and define

$$
\widetilde{E}_{n}=\rho_{n}^{-1}\left(E_{n}-E_{1, n}-E_{2, n}\right) \quad \text { and } \quad \widetilde{H}_{n}=\rho_{n}^{-1}\left(H_{n}-H_{1, n}-H_{2, n}\right) \quad \text { in } D .
$$

Then

$$
\left(\widetilde{E}_{n}, \widetilde{H}_{n}\right) \in \mathcal{N}(D)^{\perp}
$$

and

$$
\begin{cases}\nabla \times \widetilde{E}_{n}=i \mu \widetilde{H}_{n} & \text { in } D, \\ \nabla \times \widetilde{H}_{n}=-i \varepsilon \widetilde{E}_{n} & \text { in } D, \\ \nabla \times \widetilde{E}_{n} \cdot \nu=\rho_{n}^{-1} \nabla \times\left. E_{n} \cdot \nu\right|_{\text {int }} & \text { on } \partial D, \\ \nabla \times \widetilde{H}_{n} \cdot \nu=\rho_{n}^{-1} \nabla \times\left. H_{n} \cdot \nu\right|_{\text {int }} & \text { on } \partial D .\end{cases}
$$


We have

$$
\begin{aligned}
\rho_{n}^{-1} \nabla \times\left. E_{n} \cdot \nu\right|_{\text {int }} & =\rho_{n}^{-1} \nabla \times\left. E_{n} \cdot \nu\right|_{\text {ext }}+\rho_{n}^{-1} \operatorname{div}_{\Gamma} h_{1, n} \\
& =\left.i H_{n} \cdot \nu\right|_{\text {ext }}+\rho_{n}^{-1} \operatorname{div}_{\Gamma} h_{1, n} \quad \text { on } \partial D .
\end{aligned}
$$

This implies, by 4.72 ,

$$
\mu \widetilde{H}_{n} \cdot \nu=\left.H_{n} \cdot \nu\right|_{\text {ext }}-i \rho_{n}^{-1} \operatorname{div}_{\Gamma} h_{1, n} \quad \text { on } \partial D .
$$

Similarly, we have

$$
\varepsilon \widetilde{E}_{n} \cdot \nu=\left.E_{n} \cdot \nu\right|_{\text {ext }}-i \rho_{n}^{-1} \operatorname{div}_{\Gamma} h_{2, n} \quad \text { on } \partial D .
$$

Using 4.68), we derive from 4.69, 4.73), and 4.74 that

$$
\left(\varepsilon \widetilde{E}_{n} \cdot \nu, \mu \widetilde{H}_{n} \cdot \nu\right) \rightarrow\left(\left.E \cdot \nu\right|_{\text {ext }},\left.H \cdot \nu\right|_{\text {ext }}\right) \text { in } H^{-1 / 2}(\partial D) \text { as } n \rightarrow \infty \text {. }
$$

It follows from Lemma 4.8 that $\left(\left(\widetilde{E}_{n}, \widetilde{H}_{n}\right)\right)_{n}$ is bounded in $\left[L^{2}(D)\right]^{6}$. Applying Lemma 4.3 to $\left(\widetilde{E}_{n}, \widetilde{H}_{n}\right)$, one can assume that

$$
\left(\widetilde{E}_{n}, \widetilde{H}_{n}\right) \text { converges to some }(\widetilde{E}, \widetilde{H}) \in \mathcal{N}(D)^{\perp} \text { in }[H(\operatorname{curl}, D)]^{2} \text {. }
$$

Moreover, from 4.72 and 4.75, we have

$$
\begin{cases}\nabla \times \widetilde{E}=i \mu \widetilde{H} & \text { in } D, \\ \nabla \times \widetilde{H}=-i \varepsilon \widetilde{E} & \text { in } D, \\ \varepsilon \widetilde{E} \cdot \nu=\left.E \cdot \nu\right|_{\mathrm{ext}} & \text { on } \partial D, \\ \mu \widetilde{H} \cdot \nu=\left.H \cdot \nu\right|_{\mathrm{ext}} & \text { on } \partial D .\end{cases}
$$

Applying Lemma 4.5 to $(E, H)$ defined in $\mathbb{R}^{3}$ and $(\widetilde{E}, \widetilde{H})$ defined in $D$ and using 4.70, 4.71), and (4.77), we deduce that $E=H=0$ in $\mathbb{R}^{3}$, which contradicts 4.67). The proof of (4.65) is complete.

We next establish (4.66). Fix a sequence $\left(\rho_{n}\right)$ converging to 0. From 4.65), one obtains that

$$
\begin{aligned}
\left\|\left(E_{\rho_{n}}, H_{\rho_{n}}\right)\right\|_{L^{2}\left(B_{5}\right)} & \leq C\left(\left\|\theta_{\rho_{n}}\right\|_{L^{2}(D)}+\left\|h_{\rho}\right\|_{H^{-1 / 2}(\partial D)}+\rho_{n}^{-1}\left\|\operatorname{div}_{\Gamma} h_{\rho_{n}}\right\|_{H^{-1 / 2}(\partial D)}\right) \\
& \leq C .
\end{aligned}
$$

Define $\left(\widetilde{E}_{\rho_{n}}, \widetilde{H}_{\rho_{n}}\right)$ in $D$ from $\left(E_{\rho_{n}}, H_{\rho_{n}}\right)$ as in the definition of $\left(\widetilde{E}_{n}, \widetilde{H}_{n}\right)$ from $\left(E_{n}, H_{n}\right)$. Using the same arguments to obtain 4.76 , we have

$$
\left(\widetilde{E}_{\rho_{n}}, \widetilde{H}_{\rho_{n}}\right) \text { converges to }(\widetilde{E}, \widetilde{H}) \in \mathcal{N}(D)^{\perp} \text { in }[H(\operatorname{curl}, D)]^{2} \text {. }
$$

Up to a subsequence, $\left(E_{\rho_{n}}, H_{\rho_{n}}\right)$ converges to $(E, H)$ in $\left[H_{\text {loc }}\left(\operatorname{curl}, \mathbb{R}^{3}\right)\right]^{2}$ and

$$
|(E(x), H(x))|=O\left(|x|^{-2}\right) \quad \text { for large }|x| .
$$

Moreover, as in 4.77), one can show that 2.9 holds. Since the limit is unique, assertion 4.66 follows. 
We finally show how to modify the proof of 4.65 to obtain 4.63). The proof is also based on a contradiction argument and is similar to the one of (4.65). However, we denote by $\left(E_{2, n}, H_{2, n}\right)$ the projection of $\left(E_{n}, H_{n}\right)$ onto $\mathcal{N}$ (note that $E_{1, n}$ and $H_{1, n}$ might not exist in this case) and define

$$
\widetilde{E}_{n}=\rho_{n}^{-1}\left(E_{n}-E_{2, n}\right) \quad \text { in } D \quad \text { and } \quad \widetilde{H}_{n}=\rho_{n}^{-1}\left(H_{n}-H_{2, n}\right) \quad \text { in } D \text {. }
$$

Then

$$
\begin{cases}\nabla \times \widetilde{E}_{n}=i \mu \widetilde{H}_{n}+\rho_{n}^{-1} \theta_{1, n} & \text { in } D, \\ \nabla \times \widetilde{H}_{n}=-i \varepsilon \widetilde{E}_{n}+\rho_{n}^{-1} \theta_{2, n} & \text { in } D, \\ \nabla \times \widetilde{E}_{n} \cdot \nu=\rho_{n}^{-1} \nabla \times\left. E_{n} \cdot \nu\right|_{\text {int }} & \text { on } \partial D, \\ \nabla \times \widetilde{H}_{n} \cdot \nu=\rho_{n}^{-1} \nabla \times\left. H_{n} \cdot \nu\right|_{\text {int }} & \text { on } \partial D .\end{cases}
$$

Since $\left(\rho_{n}^{-1} \theta_{n}\right)_{n} \rightarrow(0,0)$ in $\left[L^{2}(D)\right]^{6}$, the sequence $\left(\left(\widetilde{E}_{n}, \widetilde{H}_{n}\right)\right)_{n}$ converges to $(\widetilde{E}, \widetilde{H})$ in $\left[L^{2}(D)\right]^{6}$. Similar to the proof of 4.65 , one also derives that $(E, H)=$ $(0,0)$ in $\mathbb{R}^{3}$. This yields a contradiction. The proof is complete.

In the proof of Lemma 4.7, we used the following lemma.

Lemma 4.8. Assume that $D$ is simply connected and $(E, H) \in \mathcal{N}(D)^{\perp}$ satisfies

$$
\nabla \times E=i \mu H \quad \text { in } D \text { and } \nabla \times H=-i \varepsilon E \quad \text { in } D \text {. }
$$

We have

$$
\|(E, H)\|_{H(\operatorname{curl}, D)} \leq C\|(\varepsilon E \cdot \nu, \mu H \cdot \nu)\|_{H^{-1 / 2}(\partial D)}
$$

for some positive constant $C$ depending only on $D, \varepsilon, \mu$.

Proof. It suffices to prove that

$$
\|(E, H)\|_{L^{2}(D)} \leq C\|(\varepsilon E \cdot \nu, \mu H \cdot \nu)\|_{H^{-1 / 2}(\partial D)} .
$$

The proof is via a standard contradiction argument. Assume that there exists a sequence $\left(\left(E_{n}, H_{n}\right)\right)_{n} \subset \mathcal{N}(D)^{\perp}$ such that

$$
\begin{gathered}
\nabla \times E_{n}=i \mu H_{n} \quad \text { in } D \text { and } \nabla \times H_{n}=-i \varepsilon E_{n} \text { in } D, \\
\left\|\left(E_{n}, H_{n}\right)\right\|_{L^{2}(D)}=1 \text { for all } n,
\end{gathered}
$$

and

$$
\left(\varepsilon E_{n} \cdot \nu, \mu H_{n} \cdot \nu\right) \rightarrow 0 \quad \text { in }\left[H^{-1 / 2}(\partial D)\right]^{2} .
$$

Applying Lemma 4.3 , one might assume that $\left(E_{n}, H_{n}\right)$ converges to some $\left(E_{0}, H_{0}\right)$ in $[H(\operatorname{curl}, D)]^{2}$. Then $\left(E_{0}, H_{0}\right) \in \mathcal{N}(D)^{\perp}$ and

$$
\begin{cases}\nabla \times E_{0}=i \mu H_{0} & \text { in } D, \\ \nabla \times H_{0}=-i \varepsilon E_{0} & \text { in } D, \\ \nabla \times E_{0} \cdot \nu=\nabla \times H_{0} \cdot \nu=0 & \text { on } \partial D .\end{cases}
$$


It follows that $\left(E_{0}, H_{0}\right) \in \mathcal{N}(D)^{\perp} \cap \mathcal{N}(D)$. Hence $\left(E_{0}, H_{0}\right)=(0,0)$ in $D$, which contradicts 4.78.

We are ready to give the following.

Proof of Theorem 2.2. In this proof, we use the same notations as in the one of Theorem 2.1. Similar to the proof of Theorem 2.1, using Lemmas 4.1 and 4.2, we have, for $R>1 / 2$,

$$
\left\|\left(E_{2, \rho}, H_{2, \rho}\right)\right\|_{L^{2}\left(B_{3 R} \backslash B_{2 R}\right)} \leq C \rho^{3}\left\|J_{\text {ext }}\right\|_{L^{2}\left(\mathbb{R}^{3} \backslash B_{2}\right)} .
$$

Involving the same method used to prove 4.59) and 4.60), however, applying 4.65) in Lemma 4.7 instead of Lemma 4.6, we have

$$
\begin{aligned}
& \left\|\hat{H}_{1, \rho} \times\left.\nu\right|_{\text {ext }}\right\|_{H^{-1 / 2}\left(\partial B_{1}\right)}+\left\|\left.\hat{E}_{1, \rho} \cdot \nu\right|_{\text {ext }}\right\|_{H^{-1 / 2}\left(\partial B_{1}\right)} \\
& \quad \leq C \rho\left\|J_{\text {ext }}\right\|_{L^{2}\left(\mathbb{R}^{3} \backslash B_{2}\right)}
\end{aligned}
$$

and

$$
\left\|\left(E_{3, \rho}, H_{3, \rho}\right)\right\|_{L^{2}\left(B_{3 R} \backslash B_{2 R}\right)} \leq C\left(\rho^{3}\left\|J_{\mathrm{ext}}\right\|_{L^{2}\left(\mathbb{R}^{3} \backslash B_{2}\right)}+\rho^{2}\left\|J_{\mathrm{int}}\right\|_{L^{2}\left(B_{1}\right)}\right) .
$$

It is clear that 2.7) follows from 4.79 and 4.81). Moreover, by Lemma 4.6. assertion 2.8 now follows from 4.80 and the fact that $\left(E_{c}, H_{c}\right)=\left(\hat{E}_{3, \rho}, \hat{H}_{3, \rho}\right)$ in $B_{1}$.

Proof of Theorem 2.3. In this proof, we use the same notations as in the one of Theorem 2.1. Similar to the proof of Theorem 2.1, using Lemmas 4.1 and 4.2, we have, for $R>1 / 2$,

$$
\left\|\left(E_{2, \rho}, H_{2, \rho}\right)\right\|_{L^{2}\left(B_{3 R} \backslash B_{2 R}\right)} \leq C \rho^{3}\left\|J_{\text {ext }}\right\|_{L^{2}\left(\mathbb{R}^{3} \backslash B_{2}\right)} .
$$

Using the same method used to prove 4.60, however, applying (4.63) in Lemma 4.7 instead of Lemma 4.6 , we have

$$
\left\|\left(E_{3, \rho}, H_{3, \rho}\right)\right\|_{L^{2}\left(B_{3 R} \backslash B_{2 R}\right)} \leq C\left(\rho^{3}\left\|J_{\text {ext }}\right\|_{L^{2}\left(\mathbb{R}^{3} \backslash B_{2}\right)}+\rho\left\|J_{\text {int }}\right\|_{L^{2}\left(B_{1}\right)}\right) .
$$

It is clear that 2.11 follows from 4.82 and 4.83.

It remains to prove 2.12). Using the linearity of the system and applying Theorem 2.2, one can assume that $J_{\text {ext }}=0$, and $J_{\text {int }}=\mathbf{E}_{0}$ for some $\left(\mathbf{E}_{0}, \mathbf{H}_{0}\right) \in$ $\mathcal{N} \backslash\{(0,0)\}$. From the definition of $\mathcal{N}$, we have

$$
\mathbf{E}_{0} \not \equiv 0 \quad \text { and } \quad \mathbf{H}_{0} \not \equiv 0 \quad \text { in } B_{1} .
$$

Note that $\left(\hat{E}_{c}, \hat{H}_{c}\right) \in\left[H_{\text {loc }}\left(\operatorname{curl}, \mathbb{R}^{3}\right)\right]^{2}$ is the unique radiating solution to the system

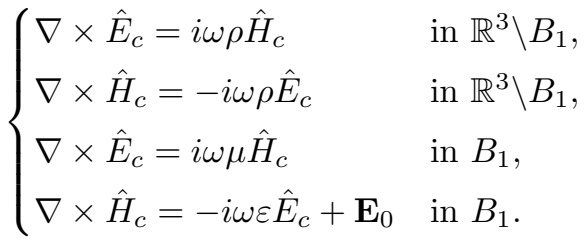


We prove 2.12 by contradiction. Assume that there exists a sequence $\left(\rho_{n}\right)_{n} \subset$ $(0,1 / 2)$ converging to 0 such that

$$
\lim _{n \rightarrow \infty} \rho_{n}\left\|\left(E_{n}, H_{n}\right)\right\|_{L^{2}\left(B_{1}\right)}=0
$$

where $\left(E_{n}, H_{n}\right) \in\left[H_{\text {loc }}\left(\operatorname{curl}, \mathbb{R}^{3}\right)\right]^{2}$ is the unique radiating solution to the system

$$
\begin{cases}\nabla \times E_{n}=i \omega \rho_{n} H_{n} & \text { in } \mathbb{R}^{3} \backslash B_{1}, \\ \nabla \times H_{n}=-i \omega \rho_{n} E_{n} & \text { in } \mathbb{R}^{3} \backslash B_{1}, \\ \nabla \times E_{n}=i \omega \mu H_{n} & \text { in } B_{1}, \\ \nabla \times H_{n}=-i \omega \varepsilon E_{n}+\mathbf{E}_{0} & \text { in } B_{1} .\end{cases}
$$

Applying Lemma 3.8 to $\left(E_{n}, H_{n}\right)$ in $B_{1}$ and using 4.84 and 4.85, we obtain

$$
\lim _{n \rightarrow \infty} \rho_{n}\left\|\left(E_{n} \times \nu, H_{n} \times \nu\right)\right\|_{H^{-1 / 2}\left(\partial B_{1}\right)}=0 .
$$

By Lemma 4.1, we have

$$
\lim _{n \rightarrow \infty} \rho_{n}\left\|\left(E_{n}, H_{n}\right)\right\|_{L^{2}\left(B_{2} \backslash B_{1}\right)}=0 .
$$

Since $\operatorname{div} E_{n}=\operatorname{div} H_{n}=0$ in $\mathbb{R}^{3} \backslash B_{1}$, we have, by Lemma 3.8 and 4.86,

$$
\lim _{n \rightarrow \infty} \rho_{n}\left\|\left(E_{n} \cdot \nu, H_{n} \cdot \nu\right)\right\|_{H^{-1 / 2}\left(\partial B_{1}\right)}=0 .
$$

It follows that

$$
\begin{aligned}
\lim _{n \rightarrow \infty} & \left\|\left(\operatorname{div}_{\Gamma}\left(E_{n} \times \nu\right), \operatorname{div}_{\Gamma}\left(H_{n} \times \nu\right)\right)\right\|_{H^{-1 / 2}\left(\partial B_{1}\right)} \\
= & \lim _{n \rightarrow \infty}\left\|\left(\left(\nabla \times E_{n}\right) \cdot \nu,\left(\nabla \times H_{n}\right) \cdot \nu\right)\right\|_{H^{-1 / 2}\left(\partial B_{1}\right)}=0 .
\end{aligned}
$$

Using the fact that $\left(\mathbf{E}_{0}, \mathbf{H}_{0}\right) \in \mathcal{N}$, we derive from 4.85 that

$$
\int_{B_{1}} \mu^{-1} \nabla \times \overline{\mathbf{E}}_{0} \cdot \nabla \times E_{n} d x-\omega^{2} \int_{B_{1}} \varepsilon \overline{\mathbf{E}}_{0} \cdot E_{n} d x=-i \omega \int_{\partial B_{1}}\left(\nu \times E_{n}\right) \cdot \overline{\mathbf{H}}_{0} d s,
$$

and

$$
\begin{array}{r}
\int_{B_{1}} \mu^{-1} \nabla \times E_{n} \cdot \nabla \times \overline{\mathbf{E}}_{0} d x-\omega^{2} \int_{B_{1}} \varepsilon E_{n} \cdot \overline{\mathbf{E}}_{0} d x \\
=-i \omega \int_{\partial B_{1}}\left(\nu \times H_{n}\right) \cdot \overline{\mathbf{E}}_{0} d s+i \omega \int_{B_{1}} \mathbf{E}_{0} \cdot \overline{\mathbf{E}}_{0} .
\end{array}
$$

Considering the imaginary part of the two identities yields

$$
\Re\left\{\int_{\partial B_{1}}\left(\nu \times H_{n}\right) \cdot \overline{\mathbf{E}}_{0} d s+\int_{\partial B_{1}}\left(\nu \times E_{n}\right) \cdot \overline{\mathbf{H}}_{0} d s\right\}=\int_{B_{1}}\left|\mathbf{E}_{0}\right|^{2}>0 .
$$

However, since $\nabla \times \mathbf{H}_{0} \cdot \nu=0$ on $\partial B_{1}$, by Lemma 3.2 there exists $\mathbf{H} \in H\left(\operatorname{curl}, B_{1}\right)$ such that

$$
\nabla \times \mathbf{H}_{0}=\nabla \times \mathbf{H} \quad \text { in } B_{1} \quad \text { and } \quad \mathbf{H} \times \nu=0 \quad \text { on } \partial B_{1}
$$


Since $\nabla \times\left(\mathbf{H}_{0}-\mathbf{H}\right)=0$ in $B_{1}$, by Lemma 3.1. there exists $\xi \in H^{1}\left(B_{1}\right)$ such that

$$
\mathbf{H}_{0}-\mathbf{H}=\nabla \xi \quad \text { in } B_{1},
$$

and hence

$$
\mathbf{H}_{0} \times \nu=\nabla \xi \times \nu \quad \text { on } \partial B_{1}
$$

We have thus

$$
\begin{aligned}
\int_{\partial B_{1}}\left(\nu \times E_{n}\right) \cdot \overline{\mathbf{H}}_{0} d s & =\int_{\partial B_{1}}\left(\nu \times E_{n}\right) \cdot \nabla \bar{\xi} d s \\
& =\int_{\partial B_{1}} \operatorname{div}_{\Gamma}\left(\nu \times E_{n}\right) \bar{\xi} d s \rightarrow 0 \quad \text { as } n \rightarrow+\infty
\end{aligned}
$$

thanks to 4.87. Similarly, we obtain

$$
\int_{\partial B_{1}}\left(\nu \times H_{n}\right) \cdot \overline{\mathbf{E}}_{0} d s \rightarrow 0 \quad \text { as } n \rightarrow+\infty .
$$

Combining 4.88-4.90, we obtain a contradiction. Hence 2.12 holds. The proof is complete.

\section{Optimality of the Degree of Visibility}

In this section, we present various settings that justify the optimality of the degree of visibility in Theorems 2.1 2.3. In what follows in this section, we assume that

$$
\varepsilon=\mu=I \quad \text { in } B_{1} .
$$

Let $h_{n}^{(1)}(n \in \mathbb{N})$ be the spherical Hankel function of first kind of order $n$ and let $j_{n}, y_{n}$ denote, respectively, its real and imaginary parts. For $-n \leq m \leq n, n \in \mathbb{N}$, denote $Y_{n}^{m}$ the spherical harmonic function of order $n$ and degree $m$ and set

$$
U_{n}^{m}(\hat{x}):=\nabla_{\partial B_{1}} Y_{n}^{m}(\hat{x}) \quad \text { and } \quad V_{n}^{m}(\hat{x}):=\hat{x} \times U_{n}^{m}(\hat{x}) \quad \text { for } \hat{x} \in \partial B_{1} .
$$

We recall that $Y_{n}^{m}(\hat{x}) \hat{x}, U_{n}^{m}(\hat{x})$, and $V_{n}^{m}(\hat{x})$ for $-n \leq m \leq n, n \in \mathbb{N}$ form an orthonormal basis of $\left[L^{2}\left(\partial B_{1}\right)\right]^{3}$.

We have the following.

Lemma 5.1. System 2.1] is non-resonant if and only if $j_{n}(\omega) \neq 0$ for all $n \geq 1$.

Proof. Assume that $j_{n}(\omega)=0$ for some $n \geq 1$. Fix such an $n$ and define, in $B_{1}$,

$$
\begin{aligned}
\mathbf{E}_{0}(x) & =j_{n}(\omega r) V_{n}^{0}(\hat{x}), \\
\mathbf{H}_{0}(x) & =\frac{n(n+1)}{i \omega r} j_{n}(\omega r) Y_{n}^{0}(\hat{x}) \hat{x}+\frac{1}{i \omega r}\left[j_{n}(\omega r)+\omega r j_{n}^{\prime}(\omega r)\right] U_{n}^{0}(\hat{x}),
\end{aligned}
$$

where $r=|x|$ and $\hat{x}=x /|x|$. Then $\left(\mathbf{E}_{0}, \mathbf{H}_{0}\right) \in \mathcal{N}$. System 2.1) is hence resonant. Conversely, assume that $j_{n}(\omega) \neq 0$ for all $n \in \mathbb{N}$. Using separation of variables (see e.g. Theorem 2.48 of Ref. 21), one can check that if $\left(\mathbf{E}_{0}, \mathbf{H}_{0}\right) \in \mathcal{N}$, then $\left(\mathbf{E}_{0}, \mathbf{H}_{0}\right)=(0,0)$ in $B_{1}$. 
The following result implies the optimality of 2.3 with respect to $J_{\text {ext }}$. For computational ease, instead of considering fields generated by $J_{\text {ext }}$, we deal with fields generated by a plane wave. In what follows, we assume that $0<\rho<1 / 2$. We have the following.

Proposition 5.1. Set $v(x):=(0,1,0) e^{i \omega x_{3}}$ for $x \in \mathbb{R}^{3}$. There exists $\omega>0$ such that

$$
\left\|E_{c}\right\|_{L^{2}\left(B_{4} \backslash B_{2}\right)} \geq C \rho^{3}
$$

for some positive constant $C$ independent of $\rho$. Here $\left(E_{c}, H_{c}\right) \in\left[H_{\mathrm{loc}}\left(\operatorname{curl}, \mathbb{R}^{3}\right)\right]^{2}$ is uniquely determined by

$$
\begin{cases}\nabla \times E=i \omega \mu_{c} H & \text { in } \mathbb{R}^{3}, \\ \nabla \times H=-i \omega \varepsilon_{c} E & \text { in } \mathbb{R}^{3},\end{cases}
$$

where $E=E_{c}+v$ and $H=H_{c}+\frac{1}{i \omega} \nabla \times v$ and by the radiation condition. Here $\left(\varepsilon_{c}, \mu_{c}\right)$ is defined by 2.1], where $(\varepsilon, \mu)$ is given in (5.1).

Proof. Let $\omega>0$ be such that $j_{1}(\omega) \neq 0$. Set

$$
\left(\mathcal{E}_{\rho}, \mathcal{H}_{\rho}\right)=\left(F_{\rho}^{-1} * E, F_{\rho}^{-1} * H\right) \quad \text { in } \mathbb{R}^{3},
$$

and define

$$
\left(\mathbf{E}_{\rho}, \mathbf{H}_{\rho}\right)=\left\{\begin{array}{cl}
\left(\mathcal{E}_{\rho}-v, \mathcal{H}_{\rho}-\frac{1}{i \omega} \nabla \times v\right) & \text { in } \mathbb{R}^{3} \backslash B_{\rho} \\
\left(\mathcal{E}_{\rho}, \mathcal{H}_{\rho}\right) & \text { in } B_{\rho} .
\end{array}\right.
$$

Set

$$
\left(\tilde{\mathbf{E}}_{\rho}, \tilde{\mathbf{H}}_{\rho}\right)=\left(\mathbf{E}_{\rho}, \mathbf{H}_{\rho}\right)(\rho \cdot) \text { and } \tilde{v}=v(\rho \cdot) \text { in } \mathbb{R}^{3}
$$

We have

$$
\begin{cases}\nabla \times \tilde{\mathbf{E}}_{\rho}=i \rho \omega \tilde{\mathbf{H}}_{\rho} & \text { in } \mathbb{R}^{3} \backslash B_{1}, \\ \nabla \times \tilde{\mathbf{H}}_{\rho}=-i \rho \omega \tilde{\mathbf{E}}_{\rho} & \text { in } \mathbb{R}^{3} \backslash B_{1}, \\ \nabla \times \tilde{\mathbf{E}}_{\rho}=i \omega \tilde{\mathbf{H}}_{\rho} & \text { in } B_{1}, \\ \nabla \times \tilde{\mathbf{H}}_{\rho}=-i \omega \tilde{\mathbf{E}}_{\rho} & \text { in } B_{1}, \\ {\left[\tilde{\mathbf{E}}_{\rho} \times \nu\right]=-\tilde{v} \times \nu, \quad\left[\tilde{\mathbf{H}}_{\rho} \times \nu\right]=-\frac{1}{i \rho \omega}(\nabla \times \tilde{v}) \times \nu} & \text { on } \partial B_{1} .\end{cases}
$$

Denote

$$
A_{\mathrm{ext}}=\left.\int_{\partial B_{1}} \tilde{\mathbf{E}}_{\rho}\right|_{\mathrm{ext}} \cdot \bar{V}_{1}^{1} d s \quad \text { and } \quad A_{\mathrm{int}}=\left.\int_{\partial B_{1}} \tilde{\mathbf{E}}_{\rho}\right|_{\mathrm{int}} \cdot \bar{V}_{1}^{1} d s .
$$

Using the transmission condition for $\tilde{\mathbf{E}}_{\rho} \times \nu$ on $\partial B_{1}$ and considering only the component with respect to $V_{1}^{1}$ for $\tilde{\mathbf{E}}_{\rho}$ (see e.g. Theorem 2.48 of Ref. 21), we have

$$
A_{\text {ext }}-A_{\text {int }}=\alpha,
$$


where

$$
\alpha=-\int_{\partial B_{1}} \tilde{v} \cdot \bar{V}_{1}^{1} d s
$$

Using the transmission condition for $\tilde{\mathbf{H}}_{\rho} \times \nu$ on $\partial B_{1}$ and considering the component with respect to $U_{1}^{1}$ for $\tilde{\mathbf{H}}_{\rho}$ (see e.g. Theorem 2.48 of Ref. 21), we have

$$
a_{\text {ext }}(\omega \rho) A_{\text {ext }}-a_{\text {int }}(\omega) A_{\text {int }}=\beta,
$$

where

$$
\begin{aligned}
& a_{\mathrm{ext}}(r)=\frac{\left(h_{1}^{(1)}(r)+r h_{1}^{(1)}(r)\right)}{-i r h_{1}^{(1)}(r)}, \\
& a_{\mathrm{int}}(r)=\frac{\left(j_{1}(r)+r j_{1}^{\prime}(r)\right)}{-i r j_{1}(r)}, \quad \text { and } \quad \beta=\alpha a_{\mathrm{int}}(\omega \rho) .
\end{aligned}
$$

Combining (5.2) and $(5.3)$ yields

$$
A_{\mathrm{ext}}=\frac{\beta-\alpha a_{\mathrm{int}}(\omega)}{a_{\mathrm{ext}}(\omega \rho)-a_{\mathrm{int}}(\omega)} .
$$

Since

$$
h_{1}^{(1)}(x)=i \frac{d}{d x} \frac{e^{i x}}{x}=\frac{\sin x-x \cos x}{x^{2}}+i \frac{x \sin x-\cos x}{x^{2}} \quad \text { for } x \in \mathbb{R},
$$

we derive that

$$
\liminf _{\rho \rightarrow 0} \rho^{-1}\left|a_{\text {ext }}(\omega \rho)-a_{\text {int }}(\omega)\right|^{-1}>0 .
$$

Since, by separation of variables (see e.g. Theorem 2.48 of Ref. 21),

$$
\left|\int_{\partial B_{1}} \tilde{v} \cdot \bar{V}_{1}^{1} d s\right|=\left|\frac{j_{1}(\omega \rho)}{j_{1}(\omega)} \int_{\partial B_{1}} v \cdot \bar{V}_{1}^{1} d s\right|
$$

we have

$$
C^{-1} \rho \leq|\alpha| \leq C \rho
$$

for some positive constant $C$ independent of $\rho$. From (5.6) and the fact that

$$
\left|a_{\text {int }}(\omega \rho)\right| \geq C \rho^{-1},
$$

we have

$$
\liminf _{\rho \rightarrow 0}\left|\beta-\alpha a_{\text {int }}(\omega)\right|>0 .
$$

Combining (5.5) and (5.7) yields

$$
\liminf _{\rho \rightarrow 0} \rho^{-1}\left|A_{\text {ext }}\right|>0 .
$$

Since, again by separation of variables,

$$
\int_{\partial B_{1}} \tilde{\mathbf{E}}_{\rho}(r \hat{x}) \cdot \bar{V}_{1}^{1}(\hat{x}) d \hat{x}=\frac{h_{1}^{(1)}(\omega \rho r)}{h_{1}^{(1)}(\omega \rho)} A_{\mathrm{ext}},
$$


and, by Lemma 3.9 ,

$$
\tilde{\mathbf{E}}_{\rho}(x / \rho)=\mathbf{E}_{\rho}(x)=\mathcal{E}_{\rho}(x)-v(x)=E_{c}(x) \quad \text { for } x \in B_{4} \backslash B_{2},
$$

we obtain the conclusion from $(5.4)$ and 5.8 .

We next show the optimality of 2.3 with respect to $J_{\text {int }}$.

Proposition 5.2. Assume that the system is non-resonant and $J_{\text {ext }}=0$ in $\mathbb{R}^{3} \backslash B_{2}$. There exists $J_{\text {int }} \in\left[L^{2}\left(B_{1}\right)\right]^{3}$ such that

$$
\liminf _{\rho \rightarrow 0} \rho^{-2}\left\|H_{C}\right\|_{L^{2}\left(B_{4} \backslash B_{2}\right)}>0 .
$$

Proof. Consider

$$
J_{\text {int }}(x)=j_{1}(\omega r) V_{1}^{1}(\hat{x}) \text { in } B_{1},
$$

where $r=|x|$ and $\hat{x}=x /|x|$. Set

$$
\mathbf{E}_{0}=J_{\text {int }} \quad \text { and } \quad \mathbf{H}_{0}=\frac{1}{i \omega} \nabla \times \mathbf{E}_{0} \quad \text { in } B_{1} .
$$

Then

$$
\begin{cases}\nabla \times \mathbf{E}_{0}=i \omega \mathbf{H}_{0} & \text { in } B_{1} \\ \nabla \times \mathbf{H}_{0}=-i \omega \mathbf{E}_{0} & \text { in } B_{1}\end{cases}
$$

Define

$$
\left(\hat{\mathbf{E}}_{\rho}, \hat{\mathbf{H}}_{\rho}\right)=\rho\left(\mathcal{E}_{\rho}, \mathcal{H}_{\rho}\right)(\rho \cdot) \quad \text { in } \mathbb{R}^{3},
$$

where $\left(\mathcal{E}_{\rho}, \mathcal{H}_{\rho}\right)$ is given in 2.13 . Then

$$
\begin{cases}\nabla \times \hat{\mathbf{E}}_{\rho}=i \rho \omega \hat{\mathbf{H}}_{\rho} & \text { in } \mathbb{R}^{3} \backslash B_{1}, \\ \nabla \times \hat{\mathbf{H}}_{\rho}=-i \rho \omega \hat{\mathbf{E}}_{\rho} & \text { in } \mathbb{R}^{3} \backslash B_{1}, \\ \nabla \times \hat{\mathbf{E}}_{\rho}=i \omega \hat{\mathbf{H}}_{\rho} & \text { in } B_{1}, \\ \nabla \times \hat{\mathbf{H}}_{\rho}=-i \omega \hat{\mathbf{E}}_{\rho}+\mathbf{E}_{0} & \text { in } B_{1} .\end{cases}
$$

We have

$$
\int_{\partial B_{1}}\left(\nu \times \hat{\mathbf{H}}_{\rho}\right) \cdot \overline{\mathbf{E}}_{0} d s-\int_{\partial B_{1}}\left(\nu \times \hat{\mathbf{E}}_{\rho}\right) \cdot \overline{\mathbf{H}}_{0} d s=\int_{B_{1}}\left|\mathbf{E}_{0}\right|^{2}>0 .
$$

We claim that

$$
\liminf _{\rho \rightarrow 0}\left|\int_{\partial B_{1}}\left(\nu \times \hat{\mathbf{E}}_{\rho}\right) \cdot \overline{\mathbf{H}}_{0} d s\right|=0 .
$$

Assuming this, we have, from (5.10),

$$
\liminf _{\rho \rightarrow 0}\left|\int_{\partial B_{1}}\left(\nu \times \hat{\mathbf{H}}_{\rho}\right) \cdot \overline{\mathbf{E}}_{0} d s\right|>0 .
$$


This implies, since $j_{1}(\omega) \neq 0$ by Lemma 5.1, that

$$
\liminf _{\rho \rightarrow 0}\left|\int_{\partial B_{1}} \hat{\mathbf{H}}_{\rho} \bar{U}_{1}^{1} d s\right|>0 .
$$

On the other hand, by the separation of variables (see e.g. Theorem 2.48 of Ref. 21),

$$
\int_{\partial B_{1}} \hat{\mathbf{H}}_{\rho}(r \hat{x}) \cdot \bar{U}_{1}^{1}(\hat{x}) d \hat{x}=\frac{h_{1}^{(1)}(\omega \rho r)+\omega \rho r h_{1}^{\prime 1}(\omega \rho r)}{r\left(h_{1}^{(1)}(\omega \rho)+\omega \rho h_{1}^{\prime 1}(\omega \rho)\right)} \int_{\partial B_{1}} \hat{\mathbf{H}}_{\rho}(\hat{x}) \cdot \bar{U}_{1}^{1}(\hat{x}) d \hat{x} .
$$

Using the fact

$$
\liminf _{\rho \rightarrow 0} \rho^{-2} \frac{1}{\left|h_{1}^{(1)}(\omega \rho)+\omega \rho h_{1}^{\prime 1}(\omega \rho)\right|}>0,
$$

and taking $r=R / \rho$ with $R \in(2,4)$ in 5.12 , we obtain

$$
\liminf _{\rho \rightarrow 0} \rho^{-3} \int_{2}^{4}\left|\int_{\partial B_{1}} \hat{\mathbf{H}}_{\rho}(R \hat{x} / \rho) \cdot \bar{U}_{1}^{1}(\hat{x}) d \hat{x}\right| d R>0 .
$$

This implies, since $H_{c}(R \hat{x})=\mathcal{H}_{\rho}(R \hat{x})=\rho^{-1} \mathbf{H}_{\rho}(R \hat{x} / \rho)$ for $R \in(2,4)$ and $\hat{x} \in \partial B_{1}$,

$$
\liminf _{\rho \rightarrow 0} \rho^{-2}\left\|H_{c}\right\|_{L^{2}\left(B_{4} \backslash B_{2}\right)}>0,
$$

which is the conclusion.

It remains to prove (5.11). Since

$$
\begin{aligned}
\mathbf{H}_{0}(x) & =\frac{1}{i \omega} \nabla \times \mathbf{E}_{0}(x) \\
& =\frac{2}{i \omega r} j_{1}(\omega r) Y_{1}^{1}(\hat{x}) \hat{x}+\frac{1}{i \omega r}\left[j_{1}(\omega r)+\omega r j_{1}^{\prime}(\omega r)\right] U_{1}^{1}(\hat{x}) \quad \text { in } B_{1},
\end{aligned}
$$

where $r=|x|$ and $\hat{x}=x /|x|$, using the separation of variables (see e.g. Theorem 2.48 of Ref. 21), we have

$$
\begin{aligned}
\liminf _{\rho \rightarrow 0}\left|\int_{\partial B_{1}}\left(\nu \times \hat{\mathbf{E}}_{\rho}\right) \cdot \overline{\mathbf{H}}_{0} d \hat{x}\right| & \leq C \liminf _{\rho \rightarrow 0}\left|\int_{\partial B_{1}} \hat{\mathbf{E}}_{\rho}(\hat{x}) \cdot \bar{V}_{1}^{1}(\hat{x}) d \hat{x}\right| \\
& =C \liminf _{\rho \rightarrow 0}\left|\frac{-i \omega \rho}{\sqrt{2}} \int_{\partial B_{1}} \hat{\mathbf{H}}_{\rho}(\hat{x})\right|_{\text {ext }} \cdot\left(\bar{Y}_{1}^{1}(\hat{x}) \hat{x}\right) d \hat{x} \mid .
\end{aligned}
$$

Since, by Lemma 4.6 .

$$
\left\|\hat{\mathbf{H}}_{\rho}\right\|_{H\left(\operatorname{curl}, B_{5}\right)} \leq C
$$

we have

$$
\liminf _{\rho \rightarrow 0}\left|\frac{-i \omega \rho}{\sqrt{2}} \int_{\partial B_{1}} \hat{\mathbf{H}}_{\rho}\right|_{\operatorname{ext}}\left(\bar{Y}_{1}^{1}(\hat{x}) \hat{x}\right) d \hat{x} \mid=0 .
$$

Thus, 5.11 follows from (5.14) and 5.15). 
We finally show the optimality of 2.11 in the case where $J_{\text {ext }} \equiv 0$ and $J_{\text {int }}$ does not satisfy the compatibility condition.

Proposition 5.3. Assume that $J_{\text {ext }}=0$ in $\mathbb{R}^{3} \backslash B_{2}$ and $j_{1}(\omega)=0$. There exists $J_{\text {int }} \in\left[L^{2}\left(B_{1}\right)\right]^{3}$ such that

$$
\left\|E_{c}\right\|_{L^{2}\left(B_{4} \backslash B_{2}\right)} \geq C \rho
$$

for some positive constant $C$ independent of $\rho$.

Proof. Define $J_{\text {int }}$ by 5.9 . We use the notations in the proof of Proposition 5.2. We have

$$
\int_{\partial B_{1}}\left(\nu \times \hat{\mathbf{H}}_{\rho}\right) \cdot \overline{\mathbf{E}}_{0} d s-\int_{\partial B_{1}}\left(\nu \times \hat{\mathbf{E}}_{\rho}\right) \cdot \overline{\mathbf{H}}_{0} d s=\int_{B_{1}}\left|\mathbf{E}_{0}\right|^{2}>0 .
$$

Since $j_{1}(\omega)=0$, it follows that

$$
\int_{\partial B_{1}}\left(\nu \times \hat{\mathbf{H}}_{\rho}\right) \cdot \overline{\mathbf{E}}_{0} d s=0 .
$$

We derive from 5.16 that ${ }^{\mathrm{d}}$

$$
\liminf _{\rho \rightarrow 0}\left|\int_{\partial B_{1}}\left(\nu \times \hat{\mathbf{E}}_{\rho}\right) \cdot \overline{\mathbf{H}}_{0} d s\right|>0 .
$$

This implies, by 5.13 ,

$$
\liminf _{\rho \rightarrow 0}\left|\int_{\partial B_{1}} \hat{\mathbf{E}}_{\rho}(\hat{x}) \cdot \bar{V}_{1}^{1}(\hat{x}) d \hat{x}\right|>0
$$

By the separation of variables (see e.g. Theorem 2.48 of Ref. 21), for $r>2$, we obtain

$$
\int_{\partial B_{1}} \hat{\mathbf{E}}_{\rho}(r \hat{x}) \cdot \bar{V}_{1}^{1}(\hat{x}) d \hat{x}=\frac{h_{1}^{(1)}(\omega \rho r)}{h_{1}^{(1)}(\omega \rho)} \int_{\partial B_{1}} \hat{\mathbf{E}}_{\rho}(\hat{x}) \cdot \bar{V}_{1}^{1}(\hat{x}) d \hat{x} .
$$

Taking $r=R / \rho$ with $R \in(2,4)$ in 5.18 , since $\lim _{\rho \rightarrow 0} \rho^{-2}\left|\frac{h_{1}^{(1)}(\omega R)}{h_{1}^{(1)}(\omega \rho)}\right|>0$, we obtain from 5.17 that

$$
\liminf _{\rho \rightarrow 0} \rho^{-2} \int_{2}^{4}\left|\int_{\partial B_{1}} \hat{\mathbf{E}}_{\rho}(R \hat{x} / \rho) \cdot \bar{V}_{1}^{1}(\hat{x}) d \hat{x}\right| d R>0 .
$$

This implies

$$
\liminf _{\rho \rightarrow 0} \rho^{-1}\left\|E_{c}\right\|_{L^{2}\left(B_{4} \backslash B_{2}\right)}>0
$$

which is the conclusion.

\footnotetext{
${ }^{\mathrm{d}}$ This is the difference between the resonant and the non-resonant cases.
} 


\section{References}

1. A. Alonso and A. Valli, Some remarks on the characterization of the space of tangential traces of $\mathrm{H}(\mathrm{rot} ; \cdots)$ and the construction of an extension operator, Manuscripta Math. 89 (1996) 159-178.

2. H. Ammari and J. C. Nedelec, Low-frequency electromagnetic scattering, SIAM J. Math. Anal. 31 (2000) 836-861.

3. H. Ammari, H. Kang, H. Lee and M. Lim, Enhancement of near-cloaking using generalized polarization tensors vanishing structures. Part I: The conductivity problem, Comm. Math. Phys. 317 (2013) 253-266.

4. H. Ammari, H. Kang, H. Lee and M. Lim, Enhancement of near-cloaking. Part II: The Helmholtz equation, Comm. Math. Phys. 317 (2013) 485-502.

5. H. Ammari, H. Kang, H. Lee, M. Lim and S. Yu, Enhancement of near cloaking for the full Maxwell equations, SIAM J. Appl. Math. 73 (2013) 2055-2076.

6. H. Ammari, M. Vogelius and D. Volkov, Asymptotic formulas for perturbations in the electromagnetic fields due to the presence of inhomogeneities of small diameter II. The full Maxwell equations, J. Math. Pures Appl. 80 (2001) 769-814.

7. G. Bao, H. Liu and J. Zou, Nearly cloaking the full Maxwell equation: Cloaking active contents with general conducting layers, J. Math. Pures Appl. 101 (2014) 716-733.

8. H. Brezis, Functional Analysis, Sobolev Spaces and Partial Differential Equations, Universitext (Springer, 2010).

9. A. Buffa, M. Costabel and D. Sheen, On traces for $H(\operatorname{curl}, \Omega)$ in Lipschitz domains, J. Math. Anal. Appl. 276 (2002) 845-867.

10. Y. Capdeboscq, On the scattered field generated by a ball inhomogeneity of constant index, Asymptot. Anal. 77 (2012) 197-246.

11. D. Colton and R. Kress, Inverse Acoustic and Electromagnetic Scattering Theory, 3rd edn. (Springer, 2013).

12. M. Costabel, A remark on the regularity of solutions of Maxwell's equations on Lipschitz domains, Math. Methods Appl. Sci. 12 (1990) 365-368.

13. Y. Deng, H. Liu and G. Uhlmann, Full and partial cloaking in electromagnetic scattering, Arch. Ration. Mech. Anal. 223 (2017) 265-299.

14. V. Girault and P. A. Raviart, Finite Element Methods for Navier-Stokes Equations, Theory and Algorithms (Springer-Verlag, 1986).

15. A. Greenleaf, Y. Kurylev, M. Lassas and G. Uhlmann, Full-wave invisibility of active devices at all frequencies, Comm. Math. Phys. 275 (2007) 749-789.

16. A. Greenleaf, M. Lassas and G. Uhlmann, On nonuniqueness for Calderon's inverse problem, Math. Res. Lett. 10 (2003) 685-693.

17. A. Greenleaf, Y. Kurylev, M. Lassas and G. Uhlmann, Improvement of cylindrical cloaking with the SHS lining, Opt. Express 15 (2007) 12717-12734.

18. R. Griesmaier and M. S. Vogelius, Enhanced approximate cloaking by optimal change of variables, Inverse Problems 30 (2014) 035014.

19. H. Haddar, P. Joly and H.-M. Nguyen, Generalized impedance boundary conditions for scattering problems from strongly absorbing obstacles: The case of Maxwell's equations, Math. Models Methods Appl. Sci. 18 (2008) 1787-1827.

20. H. Heumann and M. S. Vogelius, Analysis of an enhanced approximate cloaking scheme for the conductivity problem, Asymptot. Anal. 87 (2014) 22-246.

21. A. Kirsch and F. Hettlich, The Mathematical Theory of Time-Harmonic Maxwell's Equations, Expansion, Integral, and Variational Methods (Springer, 2015).

22. R. E. Kleinman, Far field scattering at low frequencies, Appl. Sci. Res. 18 (1968) 1-8.

23. R. V. Kohn, D. Onofrei, M. S. Vogelius and M. I. Weinstein, Cloaking via change of variables for the Helmholtz equation, Comm. Pure Appl. Math. 63 (2000) 973-1016. 
24. R. V. Kohn, H. Shen, M. S. Vogelius and M. I. Weinstein, Cloaking via change of variables in electric impedance tomography, Inverse Problems 24 (2008) 015-016.

25. M. Lassas and T. Zhou, The blow-up of electromagnetic fields in 3-dimensional invisibility cloaking for Maxwell's equations, SIAM J. Appl. Math. 76 (2016) 457-478.

26. U. Leonhardt, Optical conformal mapping, Science 312 (2006) 1777-1780.

27. H.-M. Nguyen, Cloaking via change of variables for the Helmholtz equation in the whole space, Comm. Pure Appl. Math. 63 (2010) 1505-1524.

28. H.-M. Nguyen, Approximate cloaking for the Helmholtz equation via transformation optics and consequences for perfect cloaking, Comm. Pure Appl. Math. 65 (2012) $155-186$.

29. H.-M. Nguyen, Superlensing using complementary media and reflecting complementary media for electromagnetic waves, Adv. Nonlinear Anal. 7 (2018) 449-467.

30. H.-M. Nguyen and M. S. Vogelius, Full range scattering estimates and their application to cloaking, Arch. Ration. Mech. Anal. 203 (2012) 769-807.

31. H.-M. Nguyen and M. S. Vogelius, Approximate cloaking for the wave equation via change of variables, SIAM J. Math. Anal. 44 (2012) 1894-1924.

32. H.-M. Nguyen and M. S. Vogelius, Approximate cloaking for the full wave equation via change of variables: The Drude-Lorentz model, J. Math. Pures Appl. 106 (2016) 797-836.

33. T. Nguyen and J. N. Wang, Quantitative uniqueness estimate for the Maxwell system with Lipschitz anisotropic media, Proc. Amer. Math. Soc. 140 (2012) 595-605.

34. J. B. Pendry, D. Schurig and D. R. Smith, Controlling electromagnetic fields, Science 321 (2006) 1780-1782.

35. R. Picard, On the boundary value problems of electro- and magnetostatics, Proc. Roy. Soc. Edinburgh Sect. A 92 (1982) 165-174.

36. R. Picard, On the low frequency asymptotics in electromagnetic theory, J. Reine Angew. Math. 354 (1984) 50-73.

37. M. S. Vogelius and D. Volkov, Asymptotic formulas for perturbations in the electromagnetic fields due to the presence of inhomogeneities of small diameter, M2AN Math. Model. Numer. Anal. 34 (2000) 723-748.

38. R. Weder, A rigorous analysis of high-order electromagnetic invisibility cloaks, $J$. Phys. A, Math. Theor. 41 (2008) 065207.

39. R. Weder, The boundary conditions for point transformed electromagnetic invisibility cloaks, J. Phys. A, Math. Theor. 41 (2008) 415401. 